\title{
Comparison theorems for exit times
}

\author{
Almut Burchard* and Michael Schmuckenschläger $^{\dagger}$
}

February 9, 2000. Revised December 27, 2000

\begin{abstract}
We study bounds on the exit time of Brownian motion from a set in terms of its size and shape, and the relation of such bounds with isoperimetric inequalities. The first result is an upper bound for the distribution function of the exit time from a subset of a sphere or hyperbolic space of constant curvature in terms of the exit time from a disc of the same volume. This amounts to a rearrangement inequality for the Dirichlet heat kernel. To connect this inequality with the classical isoperimetric inequality, we derive a formula for the perimeter of a set in terms of the heat flow over the boundary. An auxiliary result generalizes Riesz' rearrangement inequality to multiple integrals.
\end{abstract}

\section{Introduction}

\subsection{An inequality for exit times}

Let $\mathbb{M}_{\kappa}$ be the sphere $(\kappa>0)$, Euclidean space $(\kappa=0)$, or hyperbolic space $(\kappa<0)$ of constant curvature $\kappa$. Denote by $X_{t}$ Brownian motion on $\mathbb{M}_{\kappa}$ (see, e.g., [24, 42]). The exit time from a set $A$ is defined by

$$
T_{A}=\inf \left\{t>0: X_{t} \notin A\right\} ;
$$

it is a stopping time if $A$ is Borel measurable. We are interested in bounds on the distribution function

$$
u_{A}(t, x)=\mathbf{P}_{x}\left(T_{A}>t\right)
$$

in terms of the size and shape of $A$. We will obtain such bounds by comparing the exit time from $A$ with the exit time from a disc of the same volume.

If $A$ is a sufficiently regular open set, then $u_{A}$ solves the heat equation

$$
\partial_{t} u(t, x)=\Delta u(t, x) \quad x \in A, t>0
$$

\footnotetext{
*Department of Mathematics, University of Virginia, Charlottesville, VA 22903, burchard@virginia . edu.

${ }^{\dagger}$ Institut für Analysis und Numerik, Johannes Kepler Universität Linz, A-4040 Linz, Austria, michael.schmuckenschlaeger@telering.at.
} 
with Dirichlet boundary conditions

$$
u(t, x)=0 \quad x \in \partial A, t>0
$$

and initial values

$$
u(0, x)=1 \quad x \in A .
$$

Theorem 1 (Rearrangement for exit times on $\mathbb{M}_{\kappa}$ ) Let $A \subset \mathbb{M}_{\kappa}$ be a Borel set of finite volume, and let $A^{*}$ be an open geodesic disc of equal volume as $A$. Then, for all $t>0$, the exit time from $A$ is dominated by the exit time from $A^{*}$ in the sense that for every convex increasing function $F$,

$$
\int_{A} F\left(u_{A}(t, x)\right) d x \leq \int_{A} F\left(u_{A^{*}}(t, x)\right) d x
$$

where $d x$ denotes integration with respect to the uniform measure. In particular,

$$
\sup _{x \in A} u_{A}(t, x) \leq u_{A^{*}}\left(t, x^{*}\right),
$$

where $x^{*}$ is the center of $A^{*}$. Equality in (1.6) when $F \circ u_{A^{*}}(t, \cdot)$ is non-constant, or equality in (1.7) occurs only when there is a disc $D$ so that $A \backslash D$ has zero volume and $D \backslash A$ is polar.

The majorization statement in equation (1.6) admits many equivalent reformulations [3, 15]. It implies in particular that, unless $A$ is essentially a disc, all $L^{p}$-norms of $u_{A}$ (with $p \geq 1$ ) are strictly smaller than the corresponding norms of $u_{A^{*}}$. Another implication is that for $A \subset \mathbb{M}_{\kappa}$, the moments

$$
\mathcal{M}_{p, n}(A):=\left\|\mathbf{E}_{x}\left[T_{A}^{n}\right]\right\|_{p}
$$

are maximized, among sets of a given volume, by geodesic discs (see Corollary 3.2). Such moment inequalities were established by Aizenman and Simon [2], and by McDonald [39] who showed furthermore that discs are the only critical points of the moment functionals. We will prove (1.6) in the form

$$
\int_{B} u_{A}(t, x) d x \leq \int_{B^{*}} u_{A^{*}}(t, x) d x
$$

for any Borel set $B \subset A$. If $B$ has positive volume then equality occurs only if there is a disc $D$ so that $A \backslash D$ has zero volume, $D \backslash A$ is polar, and $B$ differs from a disc concentric with $D$ by a set of zero volume. It is an open question whether inequality (1.9) can be replaced by the stronger statement that $\left(u_{A}\right)^{*}(t, x) \leq u_{A^{*}}(t, x)$ for all $t$ and $x$, or equivalently, whether (1.6) holds for any nondecreasing, not necessarily convex, function $F$. (Such pointwise inequalities were proven for elliptic equations by Talenti [48]). The special case in (1.7) asserts the obvious fact that Brownian motion starting at some point $x \in A$ tends to leave $A$ earlier than Brownian motion starting at $x^{*}$ would leave $A^{*}$. Note that the left hand sides of (refclaim:rearr-F), (1.7), and (1.9) can be finite even when $A$ has infinite volume [18].

Inequalities analogous to (1.6) were proven in $\mathbb{R}^{n}$ by Bandle [6] and by Brock and Solynin [15], and on Gauss space by Borell [13]. The one-dimensional case was proven by Friedberg and Luttinger [26]. Closely related are geometric inequalities for the eigenvalues of the Laplacian, for 
solutions of elliptic boundary value problems [48, 7], for subharmonic functions [5], and for harmonic measure [28]. The point of Theorem 1 is the characterization of the cases of equality, which was not attempted in the above sources. In an independent development, Morpurgo has recently proved rearrangement inequalities for solutions of equations of the form

$$
\partial_{t} u-\Delta u+V(x) u=0
$$

where $V$ is a potential which is bounded below and grows at infinity [40, 41]. These results contain the analogue of inequality (1.6) for the trace of the Dirichlet heat kernel as a special case. We believe that our result remains interesting, as the proof yields a lower bound for the difference between the two sides of (1.7) and (1.9), expressed as the Wiener measure of a set of paths which we describe geometrically (Proposition 2.1).

\subsection{An inequality for multiple integrals}

The proof of Theorem 1 relies on rearrangement techniques that exploit the symmetries of the spaces $\mathbb{M}_{k}$. The key observation is that the so-called two-point rearrangements, which are simple geometric manipulations that push $A$ closer to $A^{*}$, can only increase the exit time from a set $A$ (Proposition 2.1). In this context, we call $A^{*}$ the spherical rearrangement of $A$.

For technical reasons, we do not apply rearrangement arguments directly to the proof of Theorem 1. Instead, we obtain (1.9), via a Trotter product formula, from a limit as $n \rightarrow \infty$ of rearrangement inequalities for multiple integrals

$$
\mathcal{J}\left(f_{1}, \ldots, f_{n}\right)=\int \cdots \int \prod_{1 \leq i \leq n} f_{i}\left(x_{i}\right) \prod_{1 \leq i<j \leq n} k_{i j}\left(x_{i}, x_{j}\right) d x_{1} \ldots d x_{n}
$$

where all integrations are with respect to the the uniform Riemannian measure, each $f_{i}$ is a characteristic function, and $k_{i j}$ is the heat kernel at time $t / n$ if $j=i+1$ or else identically one. Taking advantage of the fact that the heat kernel on $\mathbb{M}_{\kappa}$ is a strictly decreasing function of geodesic distance, one can use rearrangement techniques to show that the functional $\mathcal{J}$ is maximized in the obvious symmetric situation.

Theorem 2 (Rearrangement for multiple integrals on $\mathbb{M}_{\kappa}$ ) Let $\mathcal{J}$ be a functional of the form (1.11) on $\mathbb{M}_{\kappa}$, and assume that all the kernels $k_{i j}$ are nonincreasing functions of distance. Then, for any nonnegative measurable functions $f_{1}, \ldots f_{n}$ on $\mathbb{M}_{k}$ which vanish at infinity, we have

$$
\mathcal{J}\left(f_{1}, \ldots, f_{n}\right) \leq \mathcal{J}\left(f_{1}^{*}, \ldots, f_{n}^{*}\right)
$$

where $f_{1}^{*}, \ldots, f_{n}^{*}$ are the spherically decreasing rearrangements of $f_{1}, \ldots, f_{n}$ about a point $x^{*}$ of $\mathbb{M}_{\kappa}$. In particular, the left hand side is finite whenever the right hand side is finite.

Suppose that (1.12) holds with equality for a collection of functions $f_{1}, \ldots, f_{n}$, where at least two of the $f_{i}$ are non-constant, and $\mathcal{J}\left(f_{1}, \ldots, f_{n}\right)$ is finite and nonzero. Let $\Gamma_{o}$ be the graph on the vertices $i=1, \ldots, n$ which has an edge between $i$ and $j$ whenever $k_{i j}$ strictly decreases with 
distance, and assume additionally that $\Gamma_{o}$ is connected. Then the $f_{i}$ are already symmetrically decreasing about a common point, i.e., there exists an isometry $\tau$ of $\mathbb{M}_{\kappa}$ so that

$$
f_{i}=f_{i}^{*} \circ \tau \text { a.e. } \quad(i=1, \ldots, n) \text { a.e. }
$$

Spherically decreasing rearrangements will be defined at the beginning of Section 2 . The assumption that the $f_{i}$ vanish at infinity guarantees that their rearrangements exist. It is automatically satisfied if $f_{i} \in L^{p}$ for some $p>0$.

A more general version of Theorem 2 was proved independently by Morpurgo [41]. The special case of (1.12) with $n=2$

$$
\int f(x) k(x, y) g(y) d x d y \leq \int f^{*}(x) k(x, y) g^{*}(y) d x d y
$$

is known as Riesz' rearrangement inequality [44]. Here, $f$ and $g$ are again nonnegative measurable functions on $\mathbb{M}_{\kappa}$ that vanish at infinity, and $k$ is a nonincreasing function of distance. Inequality (1.12) on $\mathbb{R}^{m}$ is contained in a general inequality of Brascamp, Lieb and Luttinger which does not require the kernels on the left hand side to be decreasing functions of distance but rather allows them to be rearranged along with the functions [14]. The cases of equality are understood only when the multiple integral is in the form of a convolution [37, 17].

The basic idea of approximating the spherically decreasing rearrangement with simpler rearrangements was proposed by Steiner for a proof of the isoperimetric inequality [46]. In his work on convolution kernels on the unit circle, Ahlfors replaced Steiner symmetrization with the even simpler two-point rearrangements [1]. Friedberg and Luttinger used two-point rearrangements to prove Theorem 1 in one dimension [26]. Baernstein and Taylor [5] gave the first proof that the spherically decreasing rearrangement can be approximated in $L^{p}$-spaces by repeated two-point rearrangements and proved Riesz' rearrangement inequality on spheres. Similar rearrangements appear, as compressions, in connection with discrete isoperimetric inequalities [12, 25]. Recently, Brock and Solynin have published a comprehensive study of two-point rearrangements, or polarizations [15] (see also [29, 5, 9, 8]).

\subsection{Relation of Theorems 1 and 2 with isoperimetric inequalities}

It is well known that inequalities of the type (1.6) can be obtained via the co-area formula from isoperimetric inequalities $[51,48,7]$. We are interested in the converse direction, namely recovering isoperimetric inequalities from estimates for heat kernels. Our approach is motivated by the work of Ledoux on inequalities in Gauss space [36].

There are many definitions of the perimeter of a set, which all coincide for open sets with smooth boundary. We will show that for sufficiently regular subsets $A$ of a smooth Riemannian manifold $M$,

$$
\operatorname{Per}(A)=\lim _{t \rightarrow 0^{+}} \sqrt{\frac{\pi}{4 t}} \int_{A} 1-u_{A}(t, x) d x
$$


(Proposition 4.9). The isoperimetric inequality appears in the $t \rightarrow 0^{+}$limit of Theorem 1 in the following way: Inequality (1.6) with $F(z)=z$ is equivalent to

$$
\int_{A} 1-u_{A}(t, x) d x \geq \int_{A^{*}} 1-u_{A^{*}}(t, x) d x,
$$

since, by definition, $A^{*}$ has the same volume as $A$. If $A$ is sufficiently regular, applying (1.15) to both sides of (1.16) shows that

$$
\operatorname{Per}(A) \geq \operatorname{Per}\left(A^{*}\right)
$$

which is the isoperimetric inequality. However, information about the cases of equality is lost in the limit $t \rightarrow 0^{+}$of (1.15).

The tail of the distribution function of the exit time from $A$ decays asymptotically as Const. $e^{-\lambda_{1}(A) t}$ as $t$ becomes large, where $\lambda_{1}(A)$ is the lowest eigenvalue of the (negative) Dirichlet Laplacian on $A$. Hence inequality (1.9) implies that

$$
\lambda_{1}(A) \geq \lambda_{1}\left(A^{*}\right)
$$

which is the Faber-Krahn inequality. The lower bound for the difference between the two sides of (1.9) decays sufficiently slowly with $t$ to yield a positive lower bound for the difference $\lambda_{1}(A)-$ $\lambda_{1}\left(A^{*}\right)$ unless $A$ is essentially a disc (see Proposition 2.1 and the discussion in Section 3.3).

In summary, Theorem 1 contains the isoperimetric inequality and the Faber-Krahn inequality on $\mathbb{M}_{\kappa}$ as limiting cases. One may wonder in what form Theorem 1 could extend to general Riemannian manifolds. Can one find, for a given set $A \subset M$, a suitable disc $A^{*} \subset \mathbb{M}_{\kappa}$ whose exit time dominates the exit time from $A$ ? Is there any hope for rearrangements that can symmetrize simultaneously the manifold and the functions living on it? We offer some speculations along these lines at the end of Section 4.

Classical comparison theorems on general Riemannian manifolds due to Malliavin [38], and Debiard, Gaveau and Mazet [22] (see [42] Section IIIb and [31] Theorem 5.1) give both upper and lower bounds on the exit time from small geodesic discs in terms of curvature bounds (see Section 4). Theorem 1 is restricted to manifolds of constant curvature, but requires no assumptions on the size and shape of $A$. However, there can be no corresponding lower bound on the exit time in terms of curvature and the volume of $A$ alone, since it is always possible to make the exit time small by removing a subset of small volume from $A$. Some intriguing inequalities for Dirichlet eigenvalues in two dimensions point to possible lower bounds for the exit time in terms of the conformal class of $A[43,34]$.

The proof of (1.15) relies on the following formula.

Theorem 3 (The heat flow over the boundary as a measure of the perimeter) Let $M$ be a smooth Riemannian manifold whose curvature is uniformly bounded both above and below, and let $P^{t}$ denote the heat semigroup on $M$ at time $t \geq 0$. Then, for any Borel set $A \subset M$ be a Borel set of finite volume,

$$
\operatorname{Per}(A) \geq \varlimsup_{t \rightarrow 0^{+}} \sqrt{\frac{\pi}{t}} \int_{A^{c}} P^{t} I_{A}
$$


where $A^{c}$ is the complement of $A$. In particular, the right hand side is finite for every set of finite perimeter. If the boundary of $A$ is a twice continuously differentiable submanifold, then (1.19) holds with equality (and the limit on the right hand side exists).

Theorem 3 was proved for Gauss space by Ledoux [36]. The proof relies on the technique of estimating the heat kernel by the analogue of the Euclidean heat kernel (see, for example, [22, $21,20])$. The connection with the isoperimetric inequality is as follows: By Riesz' rearrangement inequality (1.14),

$$
\int_{A^{c}} P^{t} I_{A} \geq \int_{\left(A^{*}\right)^{c}} P^{t} I_{A^{*}}
$$

Applying (1.19) on both sides of (1.20) yields (1.17) [36].

Formula (1.15) is obtained from Theorem 3 by approximating

$$
u_{A}(t, \cdot) \approx I_{A} P^{t}\left(I_{A}-I_{A^{c}}\right) .
$$

This is exact if $A$ is a half-space in $\mathbb{M}_{\kappa}$, since then both sides of (1.21) solve the same Dirichlet problem. We will show that, for any $A \subset M$ with twice continuously differentiable boundary,

$$
\int_{A} 1-u_{A}(t, x) d x=2 \int_{A^{c}} P^{t} I_{A}+o\left(t^{1 / 2}\right) \quad \text { as } t \rightarrow 0
$$

(see the proof of Proposition 4.9). Since $P^{t}$ is self-adjoint and $P^{t} I_{A}+P^{t} I_{A^{c}} \equiv 1$, we recognize (1.22) as an integrated version of (1.21). Inserting (1.22) into Theorem 3 gives formula (1.15).

It is beyond the scope of this paper to discuss how the the right hand sides of (1.15) and (1.19) relate to the various definitions of the perimeter of a Borel set $A$ as given, for example, in [49, 16]. While the right hand side of (1.15) (with $\varlimsup$ lim in place of lim) makes sense for general Borel sets (where it may take the value $+\infty$ ), we do not know whether it has desirable semicontinuity properties analogous to (4.2).

\subsection{Outline of the paper}

The paper is organized as follows. Section 2 is devoted to two-point rearrangements. We show that the distribution function of the exit time generally increases under two-point rearrangements, and give an estimate by how much (Proposition 2.1). We then prove a discrete rearrangement inequality (Lemma 2.6) and use it to obtain Theorem 2. The results of Section 2 are combined in Section 3 to prove Theorem 1. We indicate how Theorem 1 and its proof yield corresponding inequalities for exit time moments and the trace of the Dirichlet heat kernel. In Section 4, we prove Theorem 3. The approximation (1.21) is justified, and used to prove that formula (1.15) holds at least for sufficiently regular sets (Proposition 4.9). We conclude with two conjectures regarding extensions of Theorem 1 to manifolds of non-constant curvature. 


\section{Two-point rearrangements}

\subsection{Definitions}

Let $\mathbb{M}_{\kappa}$ be the $m$-dimensional sphere, Euclidean space, or hyperbolic space of constant curvature $\kappa$, and fix a point $x^{*} \in \mathbb{M}_{\kappa}$. Denote the geodesic distance of two points $x, y \in \mathbb{M}_{\kappa}$ by $d(x, y)$. The spherical rearrangement $A^{*}$ of a set $A \subset \mathbb{M}_{\kappa}$ of finite volume is the geodesic disc centered at $x^{*}$ that has the same volume as $A$. If $f$ is a nonnegative measurable function which vanishes at infinity in the sense that all its positive level sets have finite volume, we define its spherically decreasing rearrangement $f^{*}$ to be the non-increasing function of $d\left(x, x^{*}\right)$ which is equimeasurable with $f$ and lower semicontinuous.

The spherically decreasing rearrangement can be approximated by sequences of two-point rearrangements, which are defined as follows [5, 15]. A reflection on a metric space $M$ is an isometry $\sigma$ of $M$ which satisfies

1. $\sigma^{2} x=x$ for all $x \in M$;

2. $M$ is the disjoint union of the set of fixed points $H^{o}$, and two half-spaces $H^{-}$and $H^{+}$which are exchanged by $\sigma$, that is,

$$
\begin{aligned}
& \sigma x=x \quad x \in H^{o}, \\
& \sigma H^{+}=H^{-}
\end{aligned}
$$

3. $d(x, y)<d(x, \sigma y)$ for all $(x, y) \in H^{+}$.

The two-point rearrangement of a function $f$ with respect to a reflection $\sigma$ is given by

$$
f^{\sigma}(x)= \begin{cases}\max \{f(x), f(\sigma x)\}, & x \in H^{+} \\ \min \{f(x), f(\sigma x)\}, & x \in H^{-} \\ f(x), & x \in H^{\circ} .\end{cases}
$$

If $A$ is a level set of $f$, and $A^{\sigma}$ the level set of $f^{\sigma}$ at the same height, then

$$
\begin{aligned}
A^{\sigma} \cap H^{+} & =(A \cup \sigma A) \cap H^{+} \\
A^{\sigma} \cap H^{-} & =(A \cap \sigma A) \cap H^{-} \\
A^{\sigma} \cap H^{o} & =A \cap H^{o} .
\end{aligned}
$$

Clearly, $A^{\sigma}$ has the same volume as $A$, which shows that $f^{\sigma}$ is equimeasurable with $f$.

The definition of $f^{\sigma}$ in (2.3) does not require any assumptions on $f$. Measurability and decay at infinity are needed to show that the spherically decreasing rearrangement can be approximated by repeated two-point rearrangements.

The spaces $\mathbb{M}_{\kappa}$ are characterized by large families of reflections. The reflections on a sphere, seen as embedded in $\mathbb{R}^{m+1}$, are the Euclidean reflections at hyperplanes passing through the center of the sphere. The reflections on a hyperbolic space, seen in the Poincare model as the unit disc in $\mathbb{R}^{m}$ with the hyperbolic metric, are the inversions at spheres that intersect the bounding unit sphere at right angles. In either case, there exists for each pair of points $x, y \in \mathbb{M}_{\kappa}$ a unique reflection $\sigma$ with $\sigma x=y$, and the reflections generate the entire group of isometries. 


\subsection{Reflections of Brownian paths}

Consider a Borel set $A$ of finite volume in $\mathbb{M}_{\kappa}$. Denote conditional Wiener measure on paths parametrized over $[0, t]$ which join $x$ with $y$ in $\mathbb{M}_{\kappa}$ by $\mathcal{W}_{x, y}^{t}$, and let

$$
p_{A}(t ; x, y)=\int_{A} \inf _{0 \leq s \leq t} I_{A}\left(X_{s}\right) d \mathcal{W}_{x, y}^{t}
$$

be the conditional Wiener measure of the set of all paths $X_{s}$ parametrized over $[0, t]$ which connect $x$ with $y$ in $A$. In other words, $p_{A}(t ; x, \cdot)$ is the density at time $t$ of endpoints of Brownian paths which start at $x$ and remain in $A$ up to time $t$. The associated operator $P_{A}^{t}$ has the semigroup property, and is one of the possible definitions of the Dirichlet heat kernel on $A$.

Proposition 2.1 (The difference term) Fix a reflection $\sigma$ on $\mathbb{M}_{\kappa}$ together with a choice of positive half-space $H^{+}$. Let $A, B \subset \mathbb{M}_{\kappa}$ be Borel sets, and let $A^{\sigma}$ and $B^{\sigma}$ be their two-point rearrangements. Then

$$
\int_{B^{\sigma}} u_{A^{\sigma}}(t, x) d x-\int_{B} u_{A}(t, x) d x=\int_{B} \int_{A} \mathcal{W}_{x, y}^{t}\left(E^{t}\right) d y d x,
$$

where $E^{t}$ is the event that a path does not leave $A^{\sigma}$ during $[0, t]$ and meets both $A \backslash \sigma A$ and $\sigma A \backslash A$ during some subinterval where it remains in $\mathrm{H}^{+}$.

PROOF: We write

$$
\begin{aligned}
\int_{B} u_{A}(t, x) d x & =\int_{B} \int_{A} p_{A}(t ; x, y) d y d x \\
& =\int_{H^{+}} \int_{H^{+}} \sum_{ \pm} I_{B}\left(x^{ \pm}\right) p_{A}\left(t ; x^{ \pm}, y^{ \pm}\right) I_{A}\left(y^{ \pm}\right) d y d x
\end{aligned}
$$

where $x^{ \pm}$denotes $x^{+}$or $x^{-}$,

$$
x^{+}=\left\{\begin{array}{ll}
x & x \in H^{+} \\
\sigma x & x \in H^{-}
\end{array}, \quad x^{-}=\left\{\begin{array}{ll}
\sigma x & x \in H^{+} \\
x & x \in H^{-}
\end{array},\right.\right.
$$

and the sum is over the four possible choices of $\left(x^{ \pm}, y^{ \pm}\right)$. We will show that for any pair of points $x, y \in H^{+}$and any two sets $B, C$, we have

$$
\sum_{ \pm} I_{B^{\sigma}}\left(x^{ \pm}\right) p_{A^{\sigma}}\left(t ; x^{ \pm}, y^{ \pm}\right) I_{C^{\sigma}}\left(y^{ \pm}\right)-I_{B}\left(x^{ \pm}\right) p_{A}\left(t ; x^{ \pm}, y^{ \pm}\right) I_{C}\left(y^{ \pm}\right)=\sum_{ \pm} I_{B^{\sigma}}\left(x^{ \pm}\right) \mathcal{W}_{x^{ \pm}, y^{ \pm}}^{t}\left(E^{t}\right) I_{C^{\sigma}}\left(y^{ \pm}\right)
$$

Inequality (2.6) follows from (2.10) by setting $C=A$ and integrating over $x$ and $y$.

The first term on the left hand side of (2.10) is the conditional Wiener measure of the set of paths which start in $B^{\sigma}$ at $s=0$, end in $C^{\sigma}$ at $s=t$, and join $x^{ \pm}$with $y^{ \pm}$within $A^{\sigma}$, while the second term is the corresponding quantity for $B, C$, and $A$.

We will construct a map $L$ which assigns to each path $X_{s}$ in $A$ a corresponding path $L X_{s}$ in $A^{\sigma}$. Set

$$
K=A \backslash \sigma A, \quad K^{+}=K \cap H^{+}, \quad \text { and } K^{-}=K \cap H^{-}
$$




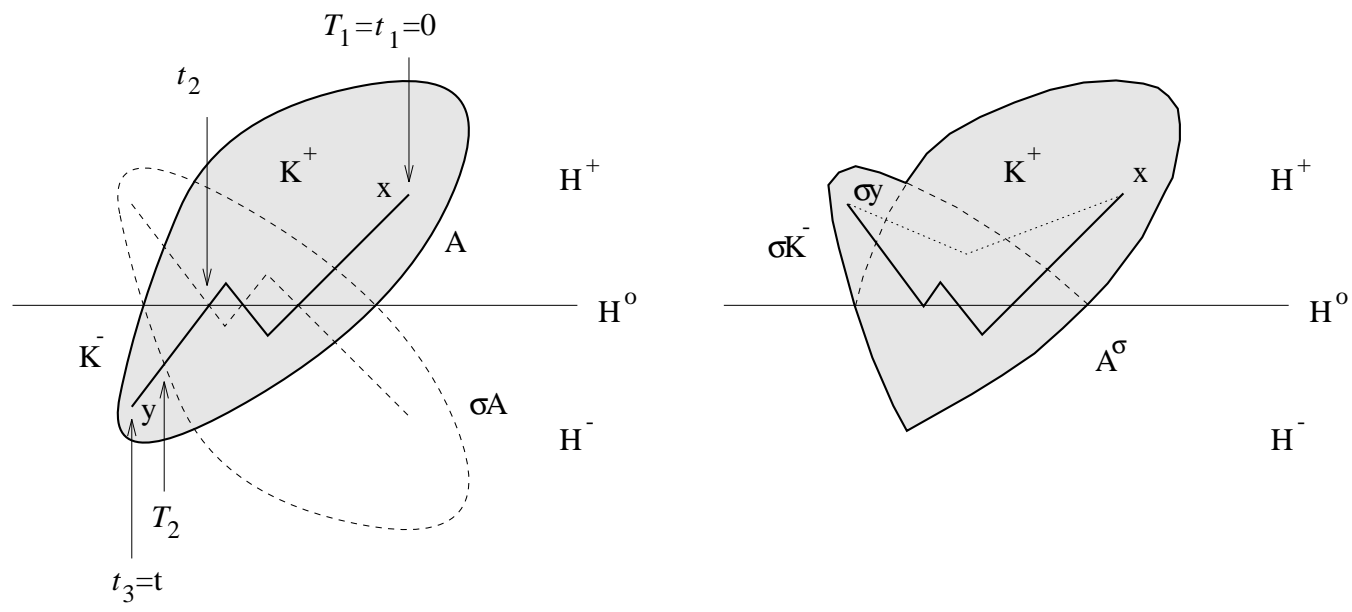

Figure 1: The reflection argument in the proof of Proposition 2.1. The figure shows a set $A$ (left, shaded), its reflection $\sigma A$ and its two-point rearrangement $A^{\sigma}$ (right). To each path $X_{s}$ in $A$ parametrized over $[0, t]$ (left, solid line) we assign a path $L X_{s}$ in $A^{\sigma}$ (solid line, right) by reflecting $X_{s}$ on certain time intervals where it hits $K^{-}$(right). The event $E^{t}$ occurs for a path if it visits both $K^{+}$and $\sigma K^{-}$without touching $H^{o}$ in-between (right, dotted line). The image of $L$ consist exactly of those paths in $A^{\sigma}$ for which $E^{t}$ does not occur.

(see Figure 1), and define a sequence of stopping times $T_{j}$ as follows.

$$
T_{1}= \begin{cases}0, & \text { if } x \in B \backslash \sigma B \\ \inf \left\{0<s \leq \min \left\{t, T_{A}\right\} \mid X_{s} \in K\right\}, & \text { if } x \in B \cap \sigma B \\ +\infty, & \text { else, }\end{cases}
$$

with the convention that the infimum of an empty set if $+\infty$. Given $T_{1}, \ldots, T_{j}$, set

$$
\begin{array}{ll}
T_{j+1}=t \quad & \text { if } X_{T_{j}} \in H^{+} \text {and } X_{t} \in(C \backslash \sigma C) \cap H^{-} \text {and } X_{s} \in A \backslash K^{-} \text {for } T_{j}<s \leq t \\
& \text { or } X_{T_{j}} \in H^{-} \text {and } X_{t} \in(C \backslash \sigma C) \cap H^{+} \text {and } X_{s} \in A \backslash K^{+} \text {for } T_{j}<s \leq t,
\end{array}
$$

otherwise set

$$
T_{j+1}= \begin{cases}\inf \left\{T_{j}<s \leq \min \left\{t, T_{A}\right\} \mid X_{s} \in K^{-}\right\} & \text {if } X_{T_{j}} \in H^{+} \\ \inf \left\{T_{j}<s \leq \min \left\{t, T_{A}\right\} \mid X_{s} \in K^{+}\right\} & \text {if } X_{T_{j}} \in H^{-}\end{cases}
$$

Denote by $N$ the number of these stopping times up to $\min \left\{t, T_{A}\right\}$. By Lemma 2.2, $N$ is almost surely finite. Let $\mathcal{S}_{n}$ be the set of sample paths in $A$ with $N=n$. (Figure 1 shows an example with $B=C=A$, and $n=2$.) By the Intermediate Value Theorem, every path $X_{s}$ in $\mathcal{S}_{n}$ with $n \geq 2$ must hit $H^{o}$ at least once between $T_{j-1}$ and $T_{j}(j=2, \ldots, n)$. Let

$$
t_{j}=\sup \left\{t \in\left(T_{j-1}, T_{j}\right) \mid X_{t} \in H^{o}\right\} \quad(j=2, \ldots, n)
$$

be the last time before $T_{j}$ that $X_{t}$ hits $H^{o}$, and set $t_{1}=0, t_{n+1}=\min \left\{t, T_{A}\right\}$. Note that though $t_{j}$ is not a stopping time (since it depends on $X_{s}$ for $s>t_{j}$ ), it is measurable with respect to the 
filtration associated with Brownian motion up to time $t$. For each path $X_{s}$ in $\mathcal{S}_{n}$, the times $t_{j}$ cut the interval $[0, t]$ into $n$ subintervals, where $X_{s}$ hits $K^{+}$and $K^{-}$on alternating subintervals. For $X_{s} \in \mathcal{S}_{n}$, define

$$
L X_{s}= \begin{cases}\sigma X_{s} & \text { for } s \in\left[t_{j}, t_{j+1}\right] \quad \text { if } X_{T_{j}} \in K^{-} \\ X_{s} & \text { else. }\end{cases}
$$

By construction, $L$ maps a path in $A$ which joins $x$ with $y$ on $[0, t]$ to a path in $A^{\sigma}$ which joins $x$ or $\sigma x$ with $y$ or $\sigma y$. Since $L$ is one-to-one and preserves Wiener measure, the left hand side of (2.10) is nonnegative. By continuity, every path in the image of $L$ meets $H^{o}$ on any interval where it hits both $K^{+}$and $\sigma K^{-}$, so

$$
L \mathcal{S}_{n} \cap E^{t}=\emptyset
$$

by definition of the event $E^{t}$. Conversely, if $E^{t}$ does not occur for a path $X_{s}$ in $A^{\sigma}$, then one can construct an inverse image $L^{-1} X_{s}$ in $A$ by reflecting the path on certain subintervals where it hits $\sigma K^{-}$. This implies (2.10) follows.

Lemma 2.2 The number $N$ of stopping times defined in the proof of Proposition 2.1 is almost surely finite.

PROOF: Fix $n>1$, and choose an index set $J \subset\{1, \ldots, n\}$. Let $\mathcal{S}_{i}$ be the set of paths with $N=i$ as in the proof of Proposition 2.1, and consider the image of $\cup_{i \geq n} \mathcal{S}_{i}$ under the maps $L_{J}$ defined by

$$
L_{J} X_{s}=\left\{\begin{array}{ll}
\sigma X_{s} & \text { for } s \in\left[t_{j-1}, t_{j}\right] \quad \text { if } j \in J \\
X_{s} & \text { else }
\end{array} .\right.
$$

Clearly, each $L_{J}$ preserves Wiener measure. Since the paths in $\cup_{i \geq n} S_{i}$ avoid $\sigma K \subset A^{\sigma} \backslash A$, the images under $L_{J}$ and $L_{J^{\prime}}$ are disjoint for $J \neq J^{\prime}$. In summary,

$$
\sum_{ \pm} \mathcal{W}_{x^{ \pm}, y^{ \pm}}^{t}\left(\cup_{j \geq n} \mathcal{S}_{j}\right) \leq 2^{-n} \sum_{ \pm} p\left(t ; x^{ \pm}, y^{ \pm}\right)
$$

where $p(t ; x, y)$ is the heat kernel on $\mathbb{M}_{\kappa}$. It follows that the expected value of $N$ is finite, and hence $N$ is finite almost surely.

\subsection{Positivity of the difference term}

Proposition 2.1 is used to analyze the cases of equality in Theorem 1 in Section 3. We will need to know that the right hand side of (2.6) is strictly positive in the cases of interest.

Proposition 2.3 Let $A \subset \mathbb{M}_{\kappa}$ be a Borel set of finite positive volume with the property that

$$
\int_{K_{1}} \int_{K_{2}} p_{A}(t ; x, y) d x d y>0
$$

for all pairs of subsets $K_{1}, K_{2} \subset A$ of positive volume. Then the right hand side of (2.6) is strictly positive unless $(A, B)=\left(A^{\sigma}, B^{\sigma}\right)$ or $(A, B)=\left(\sigma A^{\sigma}, \sigma B^{\sigma}\right)$ up to sets of zero volume. 
For an open set $A$, condition (2.20) is equivalent to requiring that $A$ is connected. Then $A^{\sigma}$ and $A^{\sigma} \cap H^{+}$are connected as well, and $E^{t}$ occurs with positive probability whenever $A^{\sigma}$ differs from both $A$ and $\sigma A$ by sets of positive volume. The next two lemmas generalize these statements to Borel sets.

Lemma 2.4 Let $A$ be a Borel set, and suppose that (2.20) holds for some $t=t_{o}>0$ and two given sets $K_{1}, K_{2} \subset$ A of finite positive volume. Then (2.20) holds for all $t>0$.

PROOF: For $t>t_{o}$,

$$
\begin{aligned}
\int_{K_{1}} \int_{K_{2}} p_{A}(t ; x, y) d x d y & =\int_{A}\left(P_{A}^{t-t_{o}} I_{K_{1}}\right)\left(P_{A}^{t_{o}} I_{K_{2}}\right) \\
\geq\left(V\left(K_{1}\right)\right)^{-1} & \left(\int_{K_{1}} \int_{K_{1}} p_{A}\left(t-t_{o} ; x, y\right) d x d y\right)\left(\int_{K_{1}} \int_{K_{2}} p_{A}\left(t_{o} ; x, y\right) d x d y(2) .22\right)
\end{aligned}
$$

where we have used the semigroup property and the self-adjointness of the heat kernel, and inserted the projection onto the characteristic function of $K_{1}$ in the second step. The second double integral on the right hand side of (2.22) is strictly positive by assumption. For the first integral, we use the positive semidefiniteness of the heat kernel to see that

$$
\left(\int_{K_{1}} \int_{K_{1}} p_{A}(s ; x, y) d x d y\right)\left(\int_{A} \int_{A} p_{A}(s ; x, y) d x d y\right) \geq\left(\int_{K_{1}} \int_{A} p_{A}(s ; x, y) d x d y\right)^{2}
$$

for all $s>0$. Since the right hand side of (2.23) is analytic and nonincreasing in $s$, it vanishes identically if it vanishes for one value of $s$. Condition (2.20) implies that it is positive at least for $s=t_{o}$, and hence for all $s>0$. Combining inequalities (2.22) and (2.23) shows that (2.20) holds for all $t \geq t_{o}$, and hence, by analyticity for all $t>0$, as claimed.

Lemma 2.5 Fix a reflection $\sigma$ on $\mathbb{M}_{\kappa}$ with positive half-space $H^{o}(\sigma)$. Let $A \subset \mathbb{M}_{\kappa}$ and $K_{1}, K_{2} \subset$ $A$ be Borel sets, with two-point rearrangements $A^{\sigma}, K_{1}^{\sigma}$ and $K_{2}^{\sigma}$. If $A, K_{1}$ and $K_{2}$ satisfy (2.20) for some $t>0$, then

$$
\int_{K_{1}^{\sigma} \cap H^{+}} \int_{K_{2}^{\sigma} \cap H^{+}} p_{A^{\sigma} \cap H^{+}}(t ; x, y) d x d y>0
$$

for all $t>0$.

PROOF: We may assume without loss of generality that $A, K_{1}$, and $K_{2}$ are symmetric under $\sigma$. Furthermore we may assume that $K_{1} \cup K_{2}=A$, otherwise we replace $K_{1}$ by

$$
K_{1} \cup\left\{x \mid \int_{K_{1}} p_{A \cap H^{+}}(t ; x, y) d y>0 \text { or } \int_{K_{1}} p_{A \cap H^{-}}(t ; x, y) d y>0\right\},
$$

and $K_{2}$ by $K_{2} \cup\left(A \backslash K_{1}\right)$. We may also assume by monotone convergence that $A$ is bounded. 
Finally, we may assume that the diameter of $A$ is as small as we please: Cover $A$ with a finite collection of balls $B_{i}$ of radius $r>0$, and let $A_{i}$ be the intersection of $A$ with the ball of radius $2 r$ concentric with $B_{i}$. Then

$$
\begin{aligned}
\int_{K_{1}} \int_{K_{2}} p_{A}(t ; x, y) d x d y & =\int I_{K_{1}}\left(P_{A}^{t} I_{K_{2}}\right) \\
\leq & \int I_{K_{1}}\left(\left(\left(I_{K_{1}}+I_{K_{2}}\right) \sum P_{A_{i}}^{t / n}\right)^{n} I_{K_{2}}\right) \\
& +\int_{K_{1}} \int_{\mathbb{M}_{\kappa}} \mathcal{W}_{x, y}^{t}\left(\left\{X_{s} \mid d\left(X_{t j / n}, X_{t(j-1) / n}\right) \geq r\right\}\right) d y d x
\end{aligned}
$$

where the last term estimates the contribution of the paths that are not contained in one of the $A_{i}$ in some interval $[t j / n, t(j-1) / n]$. Choose $n$ so large that

$$
\int_{K_{1}} \int_{\mathbb{M}_{\kappa}} \mathcal{W}_{x, y}^{t}\left(\left\{X_{s} \mid d\left(X_{t j / n}, X_{t(j-1) / n}\right) \geq r\right\}\right) d y d x<\int_{K_{1}} \int_{K_{2}} p_{A}(t ; x, y) d x d y
$$

Then

$$
\left(\left(\left(I_{K_{1}}+I_{K_{2}}\right) \sum P_{A_{i}}^{t / n}\right)^{n} I_{K_{2}}\right)>0
$$

expanding the product, we see that

$$
\int_{K_{1}} \int_{K_{2}} p_{A_{i}}(t / n ; x, y) d x d y>0
$$

for at least one value of $i$. By Lemma 2.4, $A_{i}, K_{1} \cap A_{i}$ and $K_{2} \cap A_{i}$ satisfy (2.20) for all $t>0$. Conversely, it suffices to prove the conclusion (2.24) for these three subsets.

Let now $\sigma^{\prime} \neq \sigma$ be a reflection on $\mathbb{M}_{\kappa}$ with the property that

$$
H^{+}\left(\sigma^{\prime}\right) \cap A \subset H^{+}(\sigma) \cap A
$$

(see Figure 2). In the case of Euclidean or hyperbolic space, one easily find reflections with $H^{+}\left(\sigma^{\prime}\right) \subset H^{+}(\sigma)$. On the sphere (2.31) can be satisfied when the diameter of $A$ is small enough. Set $\tau=\sigma^{\prime} \sigma$. By the continuity of Wiener measure with respect to spatial translation, we have

$$
\int_{K_{1} \cap \tau K_{1}} \int_{K_{2} \cap \tau K_{2}} p_{A \cap \tau A}(t ; x, y) d x d y>0
$$

provided that $\sigma^{\prime}$ is close to $\sigma$ in the sense that

$$
\sup _{x \in A} d\left(\sigma x, \sigma^{\prime} x\right)<\delta
$$

for some sufficiently small $\delta>0$. Note that $A \cap \tau A$ is symmetric under $\sigma^{\prime}$, since $A$ is symmetric under $\sigma$. 


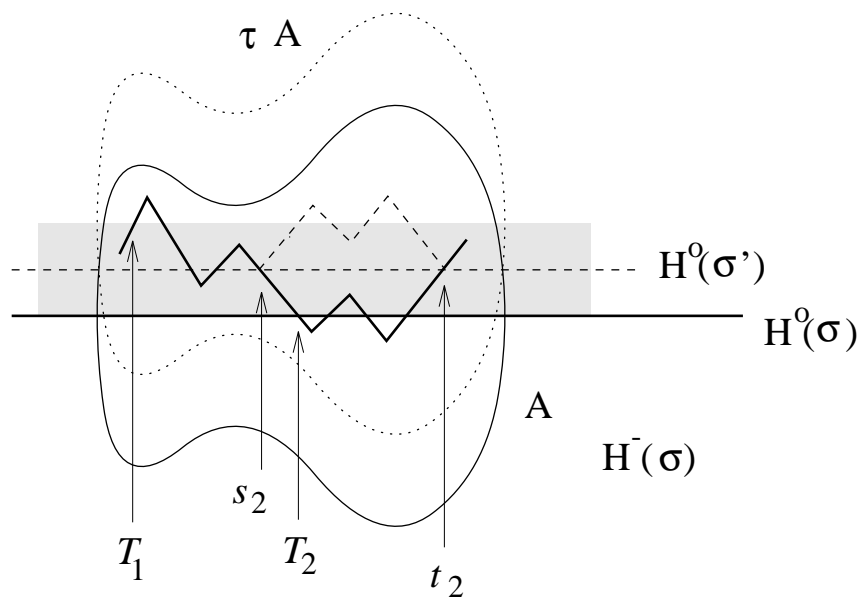

Figure 2: The reflection argument in the proof of Lemma 2.5. $A$ is symmetric under $\sigma$, and $A \cap \tau A$ is symmetric under $\sigma^{\prime}$ with $H^{+}\left(\sigma^{\prime}\right) \cap A \subset H^{+}(\sigma) \cap A$. A path in $A \cap \tau A$ (solid polygon) is mapped to a path in $H^{+}(\sigma) \cap A$ by reflecting it with $\sigma^{\prime}$ (dotted polygon) on certain subintervals where it meets $H^{-}(\sigma)$.

Similar to the proof of Proposition 2.1, we construct a transformation $L$ which maps paths in $A \cap \tau A$ to paths in $A \cap H^{+}(\sigma)$ by reflecting them at $H^{o}\left(\sigma^{\prime}\right)$ on subintervals where they meet $H^{-}(\sigma)$. Define two sequences of stopping times by

$$
\begin{aligned}
& T_{1}=\inf \left\{0<s \leq \min \left\{t, T_{A}\right\} \mid X_{s} \in H^{o}(\sigma) \cup \tau H^{o}(\sigma)\right\}, \\
& t_{i}=\inf \left\{t_{i}<s \leq \min \left\{t, T_{A}\right\} \mid X_{s} \in H^{o}\left(\sigma^{\prime}\right)\right\}, \\
& T_{i+1}=\inf \left\{t_{i}<s \leq \min \left\{t, T_{A}\right\} \mid X_{s} \in H^{o}(\sigma) \cup \tau H^{o}(\sigma)\right\},
\end{aligned}
$$

with the convention that the infimum of an empty set is $\infty$. For $i \geq 1$, set

$$
s_{i}=\sup \left\{0<s<T_{i} \mid X_{s} \in H^{o}\left(\sigma^{\prime}\right)\right\},
$$

with the convention that the supremum of an empty set is 0 . For $J \subset\{1, \ldots, n\}$, let $\mathcal{S}_{n, J}$ be the set of paths in $C$ with $T_{n} \leq t \leq T_{A}$ but $T_{n+1}>t$ and such that $X_{T_{j}} \in H^{o}(\sigma)$ exactly when $j \in J$. (Figure 2 shows an example with $n=2$ and $J=\{2\} \subset\{1,2\}$.)

For $X_{s} \in \mathcal{S}_{n, J}$, define

$$
L X_{s}= \begin{cases}\sigma^{\prime} X_{s} & \text { for } s \in\left[s_{j}, t_{j}\right] \text { with } j \in J \\ X_{s} & \text { else. }\end{cases}
$$

Although $L$ is clearly not one-to-one, its restriction to each of the $\mathcal{S}_{n, J}$ is measure-preserving and one-to-one onto $\mathcal{S}_{n, \emptyset}$. Furthermore, paths in $A \cap \tau A$ are mapped to paths in $A \cap H^{+}(\sigma)$ by (2.31). Since the paths joining $K_{1}$ with $K_{2}$ in $A$ form a set of positive Wiener measure, so do their images under $L$. This completes the proof.

Proof of Proposition 2.3 Suppose that $A^{\sigma}$ differs from both $A$ and from $\sigma A$ by sets of positive volume. Then $K^{+}$and $\sigma K^{-}$have positive volume. Applying (2.10) (with $B=B^{\sigma}=K^{+}$, 
$C=\sigma C^{\sigma}=K^{-}$) to assumption (2.20) implies that

$$
\int_{K^{+}} \int_{\sigma K^{-}} p_{A^{\sigma}}(t ; x, y) d x d y \geq \int_{K^{+}} \int_{K^{-}} p_{A}(t ; x, y) d x d y>0,
$$

and hence, by Lemma 2.5 ,

$$
\int_{K^{+}} \int_{\sigma K^{-}} p_{A^{\sigma} \cap H^{+}}(t ; x, y) d x d y>0
$$

for all $t>0$. It follows with the semigroup property that

$$
\mathcal{W}_{x, y}^{t}\left(E^{t}\right) \geq \int_{K^{+}} \int_{\sigma K^{-}} p_{A^{\sigma}}\left(t / 3 ; x, x_{1}\right) p_{A^{\sigma} \cap H^{+}}\left(t / 3 ; x_{1}, x_{2}\right) p_{A^{\sigma}}\left(t / 3 ; x_{2}, y\right) d x_{1} d x_{2}>0 .
$$

We conclude that the right hand side of (2.10) can vanish only when either both terms on the left hand side of (2.10) vanish, or $A=A^{\sigma}$ (up to a set zero volume), $x^{+} \in B, y^{+} \in C$, or $A=\sigma A^{\sigma}$ (up to a set of zero volume), $x^{+} \in \sigma B, y^{+} \in \sigma C$. Integrating over $x \in B^{\sigma}, y \in C^{\sigma}$ with $C=A$ gives the claim.

\subsection{Rearrangements for multiple integrals}

It is tempting to try to prove Theorem 1 directly from Proposition 2.1 by approximating the spherically decreasing rearrangement with a sequence of two-point rearrangements. The difficulty is that the exit time does not depend continuously on $A$ with respect to symmetric difference. We avoid this issue by using rearrangement methods only to prove Theorem 2 , and then taking an appropriate limit to get (1.9).

We first prove a discrete version of Theorem 2. Consider the two-point space $\{+,-\}$, with the metric defined by $d(+,-)=1$. The map $\sigma$ that exchanges + and - is a reflection, with no fixed points, and $H^{+}=\{+\}$and $H^{-}=\{-\}$as the positive and negative half-spaces. For any function $\phi$ on $\{+,-\}$, let $\phi^{\sigma}$ be the corresponding two-point rearrangement of $f$ :

$$
\phi^{\sigma}(+)=\max \{\phi(+), \phi(-)\}, \quad \phi^{\sigma}(-)=\min \{\phi(+), \phi(-)\} .
$$

Lemma 2.6 Let $\phi_{1}, \ldots, \phi_{n}$ be nonnegative functions on the set $\{+,-\}$. For each pair ij, let $k_{i j}\left(\varepsilon, \varepsilon^{\prime}\right)$ be a decreasing function of the distance between $\varepsilon$ and $\varepsilon^{\prime}$, i.e.,

$$
k_{i j}\left(\varepsilon, \varepsilon^{\prime}\right)=a_{i j}+b_{i j} I_{\varepsilon=\varepsilon^{\prime}}
$$

with $a_{i j}, b_{i j} \geq 0$. Consider the function

$$
J\left(\phi_{1}, \ldots, \phi_{n}\right):=\sum_{ \pm} \prod_{1 \leq i \leq n} \phi_{i}\left(\varepsilon_{i}\right) \prod_{1 \leq i<j \leq n} k_{i j}\left(\varepsilon_{i}, \varepsilon_{j}\right)
$$

Then

$$
J\left(\phi_{1}, \ldots, \phi_{n}\right) \leq J\left(\phi_{1}^{\sigma}, \ldots, \phi_{n}^{\sigma}\right)
$$


Suppose that both sides of (2.42) take the same positive value for $\phi_{1}, \ldots, \phi_{n}$. Let $\Gamma_{o}$ be the graph on the vertices $i=1, \ldots, n$ formed by the edges $i j$ with $b_{i j}>0$, and assume additionally that $\Gamma_{o}$ is connected. Then either

(i) $\phi_{i}=\phi_{i}^{\sigma}$ for $i=1, \ldots, n$, or

(ii) $\phi_{i}=\phi_{i}^{\sigma} \circ \sigma$ for $i=1, \ldots, n$.

PROOF: We write

$$
J\left(\phi_{1}, \ldots, \phi_{n}\right)=\sum_{\Gamma} K_{\Gamma} \prod_{\mathcal{C} \subset \Gamma} \Phi_{\mathcal{C}}
$$

where $\Gamma$ runs over all graphs on the vertices $i=1, \ldots, n$, the index $\mathcal{C}$ runs over the connected components of $\Gamma$,

$$
K_{\Gamma}=\prod_{i j \notin e(\Gamma)} a_{i j} \prod_{i j \in e(\Gamma)} b_{i j}, \quad \Phi_{\mathcal{C}}=\prod_{i \in \mathcal{C}} \phi_{i}(+)+\prod_{i \in \mathcal{C}} \phi_{i}(-),
$$

and $e(\Gamma)$ is the set of edges of $\Gamma$. Defining $\Phi_{\mathcal{C}}^{\sigma}$ accordingly, we may factor

$\Phi_{\mathcal{C}}^{\sigma}-\Phi_{\mathcal{C}}=\left[\prod_{i \in \mathcal{C}: \phi_{i}(+) \geq \phi_{i}(-)} \phi_{i}(+)-\prod_{i \in \mathcal{C}: \phi_{i}(+) \geq \phi_{i}(-)} \phi_{i}(-)\right] \cdot\left[\prod_{i \in \mathcal{C}: \phi_{i}(+)<\phi_{i}(-)} \phi_{i}(-)-\prod_{i \in \mathcal{C}: \phi_{i}(+)<\phi_{i}(-)} \phi_{i}(+)\right]$,

which is clearly nonnegative. It follows from (2.43) that $J$ does not decrease under rearrangement. Moreover,

$$
J\left(\phi_{1}^{\sigma}, \ldots, \phi_{n}^{\sigma}\right)-J\left(\phi_{1}, \ldots, \phi_{n}\right) \geq K_{\Gamma_{o}}\left(\Phi_{\{1, \ldots, n\}}^{\sigma}-\Phi_{\{1, \ldots, n\}}\right),
$$

where $\Gamma_{o}$ is the connected graph appearing in the statement of the lemma.

To see the claim about the cases of equality, note that the right hand side of (2.45) is positive unless one of the factors vanishes. If none of the $k_{i j}$ or $\phi_{i}$ vanishes identically, then $K_{\Gamma_{o}}$ is positive, and the right hand side of (2.46) vanishes only if $\phi_{i}(+)-\phi_{i}(-)$ does not change sign as $i=$ $1, \ldots, n$.

Lemma 2.7 (Two-point rearrangement inequality) Let $\mathcal{J}$ be a functional of the form (1.11) on $\mathbb{M}_{\kappa}$, where for each pair $i j$, the kernel $k_{i j}(x, y)$ is a nonincreasing function of the distance between $x$ and $y$. Let $\sigma$ be a reflection, with a choice of positive half-space $H^{+}$. Then, for any collection of nonnegative measurable functions $f_{1}, \ldots, f_{n}$ on $\mathbb{M}_{\kappa}$, we have

$$
\mathcal{J}\left(f_{1}, \ldots, f_{n}\right) \leq \mathcal{J}\left(f_{1}^{\sigma}, \ldots, f_{n}^{\sigma}\right)
$$

Suppose that (2.47) holds with equality for some functions $f_{1}, \ldots, f_{n}$, where the value of the right hand side is finite and positive, $n>1$, and at least two of the $f_{i}$ are non-constant. Let $\Gamma_{o}$ be the graph on the vertices $i=1, \ldots, n$ which has an edge between $i$ and $j$ whenever the kernel $k_{i j}$ is strictly decreasing with distance, and assume additionally that $\Gamma_{o}$ is connected. Then either 
(i) $f_{i}=f_{i}^{\sigma}$ for all $i$, or

(ii) $f_{i}=f_{i}^{\sigma} \circ \sigma$ for all $i$.

PROOF: Rewrite $\mathcal{J}$ as an integral over the positive half-space $H^{+}$:

$$
\mathcal{J}\left(f_{1}, \ldots, f_{n}\right)=\int_{H^{+}} \ldots \int_{H^{+}} \sum_{ \pm} \prod_{1 \leq i \leq n} f_{i}\left(x_{i}^{ \pm}\right) \prod_{1 \leq i<j \leq n} k_{i j}\left(x_{i}^{ \pm}, x_{j}^{ \pm}\right) d x_{1} \ldots d x_{n},
$$

where the $x_{i}^{ \pm}$are as in (2.9). For every fixed choice of $x_{1}, \ldots, x_{n}$ in $H^{+}$, the integrand is of the form (2.41), with

$$
\begin{array}{ll}
\phi_{i}(+)=f\left(x_{i}^{+}\right), & a_{i j}=k_{i j}\left(x_{i}^{+}, x_{j}^{-}\right) \\
\phi_{i}(-)=f\left(x_{i}^{-}\right) & b_{i j}=k_{i j}\left(x_{i}^{+}, x_{j}^{+}\right)-k_{i j}\left(x_{i}^{+}, x_{j}^{-}\right)
\end{array} .
$$

It follows directly from Lemma 2.6 that inequality (2.47) holds as claimed. Moreover, equality occurs only if for each collection of points $x_{1}, \ldots, x_{n}$, the difference $f\left(x_{i}^{+}\right)-f\left(x_{i}^{-}\right)$does not change sign as $i=1, \ldots, n$. But then the sign cannot change either if the $x_{i}$ are varied, which proves the claim about the cases of equality.

Proof OF THEOREM 2 We want to show that functionals of the form (1.11) can only increase under spherically decreasing rearrangement. Suppose that $f_{1}, \ldots, f_{n}$ are nonnegative measurable functions that vanish at infinity. By monotone convergence, we may assume that the $f_{i}$ bounded and integrable, and that $\mathcal{J}\left(f_{1}, \ldots, f_{n}\right)$ is finite. By a result of Brock and Solynin ([15], Theorem 6.1), there exists a sequence of reflections $\left\{\sigma_{j}\right\}_{j \geq 1}$ so that

$$
f^{\sigma_{1} \ldots \sigma_{j}} \rightarrow f^{*} \quad(j \rightarrow \infty)
$$

uniformly in $L^{1}$ whenever $f \in L^{1}$. It follows from Lemma 2.7 that

$$
\mathcal{J}\left(f_{1}, \ldots, f_{n}\right) \leq \mathcal{J}\left(f_{1}^{\sigma_{1} \ldots \sigma_{j}}, \ldots, f_{n}^{\sigma_{1} \ldots \sigma_{j}}\right) \rightarrow \mathcal{J}\left(f_{1}^{*}, \ldots, f_{n}^{*}\right) \quad(j \rightarrow \infty),
$$

which proves the inequality.

Equality in (1.12) implies, by (2.50), that $\mathcal{J}$ does not increase under any two-point rearrangement of the $f_{i}$. By Lemma 2.7, for every $\sigma$ and every collection of points $x_{1}, \ldots x_{n}$, either $f_{i}^{\sigma}\left(x_{i}\right)=f_{i}\left(x_{i}\right)$ for all $i$, or $f_{i}^{\sigma}\left(x_{i}\right)=f_{i}\left(\sigma x_{i}\right)$ for all $i$. Varying the $x_{i}$, we see that either $f_{i}^{\sigma}=f_{i}$ for all $i$, or $f_{i}^{\sigma}=f_{i} \circ \sigma$ for all $i$. Lemma 2.8 (proved below) shows that each $f_{i}$ is already spherically decreasing about some point $x_{i}$ of $\mathbb{M}_{\kappa}$. Using Lemma 2.7 once more, we see that all the points $x_{i}$ must coincide.

The following lemma strengthens Lemma 6.3 of [15], which says that $f=f^{*}$, if and only if $f=f^{\sigma}$ whenever $x^{*}$ is contained in the positive half-space $H^{+}(\sigma)$. 
Lemma 2.8 Let $f$ be a measurable function on $\mathbb{M}_{\kappa}$ and assume that $|f|$ vanishes at infinity. If for every reflection $\sigma$, either $f^{\sigma}=f$ (a.e.) or $f^{\sigma}=f \circ \sigma$ (a.e.), then $f$ is either spherically decreasing or spherically increasing about some point of $\mathbb{M}_{\kappa}$ (possibly after a change on a set of measure zero).

PROOF: We consider only the case where $\mathbb{M}_{\kappa}$ is a hyperbolic space of dimension $m$, as the case of the sphere is similar. Without loss of generality, $f$ is bounded and integrable; otherwise we replace it by $G \circ f$, where $G: \mathbb{R} \rightarrow \mathbb{R}$ is strictly increasing, bounded, and sufficiently small near 0 .

Fix a reflection $\sigma$ a positive half-space $H^{+}(\sigma)$, and consider

$$
\delta(\sigma)=\int_{H^{+}(\sigma)} f(x)-f(\sigma x) d x
$$

By assumption, $f-f \circ \sigma$ does not change signs in each of the two half-spaces $H^{+}(\sigma)$ and $H^{-}(\sigma)$. It follows that

$$
\delta(\sigma)>0 \Longrightarrow f^{\sigma}=f \text { (a.e.), } \quad \delta(\sigma)<0 \Longrightarrow f^{\sigma}=f \circ \sigma \text { (a.e.). }
$$

By continuity of the integral with respect to translations,

$$
\delta(\sigma)=0 \Longleftrightarrow f=f \circ \sigma \text { (a.e.) }
$$

that is, $f$ is symmetric under $\sigma$.

For $m=1$, we view $\mathbb{M}_{\kappa}$ as the line segment $(-1,1)$ with the hyperbolic metric. Each point $\alpha \in(-1,1)$ determines a (hyperbolic) reflection having that point as a fixed point. We specify the interval to the right as the positive half-space. The integrability of $f$ implies that $\delta(\sigma) \rightarrow \pm \int f$ as the fixed point approaches $\mp 1$. By the intermediate value theorem, there exists a reflection $\sigma_{1}$ with $\delta\left(\sigma_{1}\right)=0$. Then $=f \circ \sigma_{1}$ by (2.53). Using the isometries of the hyperbolic space, we assume that $\sigma_{1}$ is the Euclidean reflection at the origin, and $f$ is even. If $f$ is symmetric under a sequence of reflections $\sigma_{j}$ with fixed points $\alpha_{j} \rightarrow 0$, but with $\alpha_{j} \neq 0$, then $f$ is constant (a.e.). Otherwise, $\delta(\sigma)$ has a definite sign (say, $\delta(\sigma)>0$ ) whenever its fixed point is negative and sufficiently close to the origin. By (2.53), $f^{\sigma}=f$, that is, $f(\sigma x) \leq f(x)$ for all $x>0$. Let $y>x>0$, and let $\sigma$ be the reflection mapping $x$ to $-y$. The fixed point of $\sigma$ lies to the left of the origin. It follows that

$$
f(x)-f(y)=f(x)-f(-y)=f(x)-f(\sigma x) \geq 0 \text { (a.e.) }
$$

at least when $y>x$ is close enough to $x$. We conclude that $f$ is increasing on $[0,1)$. This completes the proof in dimension $m=1$.

For $m>1$, we view $m$-dimensional hyperbolic space as the unit disc in $\mathbb{R}^{m}$ with the Poincaré metric. A reflection is given by a sphere which intersects the boundary of the disc at a right angle, together with a choice of a positive half-space. Consider the family of such spheres with centers on the $x_{1}$-axis. The intermediate value theorem implies as in the case $m=1$ that the family contains a reflection $\sigma_{1}$ with $\delta\left(\sigma_{1}\right)=0$. Using the symmetries of hyperbolic space, we may assume that $\sigma_{1}$ is the Euclidean reflection at the hyperplane $x_{1}=0$. Repeating the argument with families of spheres 
centered on the other coordinate axes yields a collection of $m$ commuting reflections $\sigma_{1}, \ldots, \sigma_{m}$, which we may take to be the Euclidean reflections at the coordinate axes. Therefore, $\delta(\sigma)=0$ for any reflection at a hyperplane through the origin. It follows that $f$ is rotationally symmetric. The proof is completed by using the one-dimensional case to show that the restriction of $f$ to a line segment passing through the center of the unit disc depends monotonically on the distance from the center.

\section{Exit times and Dirichlet problems}

\subsection{Trotter products}

We mentioned in the introduction that for a sufficiently regular open set $A$, the distribution function of the exit time $u_{A}(t, x)$ solves the heat equation (1.3)-(1.5) on $A$ with Dirichlet boundary conditions. There are several meaningful definitions of Dirichlet boundary conditions which all coincide for sufficiently regular open sets, but may differ for more general measurable sets.

The kernel $p_{A}(t ; x, y)$ was defined in (2.5) as the conditional Wiener measure of the set of all paths on $[0, t]$ connecting $x$ with $y$ in $A$. The corresponding heat semigroup $P_{A}^{t}$ is both positivity preserving and positive definite on $H_{o}^{1}(A)$ (the closure of $C_{o}^{\infty}(A)$ in $H^{1}$ ), and vanishes on its complement.

Following Stroock [47], we define the first penetration time of $X_{t}$ into the complement of $A$ by

$$
\tilde{T}_{A}=\inf \left\{t>0: \int_{0}^{t} I_{A}\left(X_{s}\right)<t\right\} \text {. }
$$

Then $\tilde{T}_{A}$ is a stopping time. Its distribution function

$$
\tilde{u}_{A}(t, x)=\mathbf{P}_{x}\left(\tilde{T}_{A}>t\right)
$$

solves the heat equation (1.3)-(1.5) in the sense that

$$
\tilde{u}_{A}(t, x)=\int \tilde{p}_{A}(t ; x, y) I_{A}(y) d y
$$

where $\tilde{p}_{A}(t ; x, y)$ is the kernel of the Trotter product

$$
\tilde{P}_{A}^{t}=\lim _{n \rightarrow \infty}\left(I_{A} P^{t / n}\right)^{n}
$$

and $I_{A}$ is the indicator function of $A$ (see [30]). The existence of the limit in the strong $L^{2}$-sense is guaranteed by a theorem of Kato [32], since both multiplication with $I_{A}$ and the heat semigroup $P^{t}$ define contraction semigroups on $L^{2}\left(\mathbb{M}_{\kappa}\right)$. The semigroup $\tilde{P}_{A}^{t}$ defined by (3.4) is positivity preserving and positive definite on the intersection of $H^{1}\left(\mathbb{M}_{\kappa}\right)$ with the set of $L^{2}$-functions supported on $A$ (which generally contains $H_{o}^{1}(A)$ ), and vanishes on its orthogonal complement. We note that $\tilde{P}_{A}^{t}$ is another candidate for the Dirichlet heat semigroup on $A$. In contrast with $p_{A}(t ; x, y)$ and 
$u_{A}(t, x)$, the functions $\tilde{u}_{A}(t, x)$ and $\tilde{p}_{A}(t ; x, y)$ are not affected if $A$ is changed by a set of zero volume.

Since, by definition, $\tilde{T}_{A} \geq T_{A}$ for any Borel set $A$, the inequality

$$
u_{A}(t, x) \leq \tilde{u}_{A}(t, x)
$$

holds for all $t>0$ and all $x, y \in \mathbb{M}_{\kappa}$. If $A$ is an open set with smooth boundary, then $\tilde{P}_{A}^{t}$ coincides with $P_{A}^{t}$ as a semigroup on $L^{2}\left(\mathbb{M}_{\kappa}\right)$ and (3.5) holds with equality a.e. . In particular

$$
\tilde{u}_{A^{*}}=u_{A^{*}}
$$

so that an upper bound for $\tilde{u}_{A}$ such as

$$
\int_{B} \tilde{u}_{A}(t, x) d x \leq \int_{B^{*}} \tilde{u}_{A^{*}}(t, x) d x
$$

implies the corresponding upper bound (1.9) for $u_{A}$. The point is that the Trotter formula (3.4) exhibits (3.7) as a limit of inequalities of the form considered in Theorem 2.

On the other hand, let $\tilde{A}$ be the set of all points of Lebesgue density one of $A$. By a theorem of Stroock [47], any path that hits the complement of $\tilde{A}$ almost surely spends a positive amount of time outside $\tilde{A}$, so that

$$
\tilde{u}_{A}=u_{\tilde{A}}
$$

Since $\tilde{A}$ differs from $A$ by a set of zero volume, one can see (3.7) as a special case of (1.9).

Equation (2.6) for $\tilde{A}$ implies that

$$
u_{\tilde{A}^{\sigma}} \leq \tilde{u}_{A^{\sigma}}
$$

since every point of $\tilde{A}^{\sigma}$ has density one. In fact,

$$
\int_{B^{\sigma}} \tilde{u}_{A^{\sigma}}(t, x) d x-\int_{B} \tilde{u}_{A^{\sigma}}(t, x) d x \geq \int_{B} \int \mathcal{W}_{x, y}^{t}\left(\tilde{E}^{t}\right) d y d x,
$$

where $\tilde{E}^{t}$ is the event that a path does not penetrate into the complement of $A^{\sigma}$ during $[0, t]$ and spends positive time in both $\sigma A \backslash A$ and $A \backslash \sigma A$ during some subinterval of $[0, t]$ where it remains in $H^{+}$. Alternately, this can be shown by applying Lemma 2.7 to Trotter's formula, and estimating the contribution of various sets of paths with Varadhan's inequality (4.61) for the small-time behavior of the heat kernel.

\subsection{Proof of Theorem 1}

The following lemma allows to reformulate (1.6) in the form (1.9). It is due to Alvino, Trombetti, and Lions ([3], Proposition 2.1).

Lemma 3.1 Let $f$ and $g$ be nonnegative measurable functions on $\mathbb{M}_{\kappa}$ that vanish at infinity, and suppose that $g$ is symmetrically decreasing about $x^{*} \in \mathbb{M}_{\kappa}$. The following statements are equivalent. 


\section{For every Borel set B:}

$$
\int_{B} f(x) d x \leq \int_{B^{*}} g(x) d x,
$$

with equality only if B has zero volume.

2. For every nonnegative convex function $F$ for which the integrals are finite,

$$
\int F(f(x)) d x \leq \int F(g(x)) d x,
$$

with equality only if $F \circ g \equiv$ const..

Proof of Theorem 1 Let $A, B \subset \mathbb{M}_{\kappa}$ be Borel sets of finite volume. Then

$$
\begin{aligned}
\int_{B} \tilde{u}_{A}(t & , x) d x=\int I_{B}(x) \tilde{p}_{A}(t, x, y) I_{A}(y) d y d x \\
& =\lim _{n \rightarrow \infty} \int \ldots \int I_{B}\left(x_{1}\right) p\left(t / n ; x_{1}, x_{2}\right) I_{A}\left(x_{2}\right) \ldots p\left(t / n ; x_{n-1}, x_{n}\right) I_{A}\left(x_{n}\right) d x_{1} \ldots d x \\
& \leq \lim _{n \rightarrow \infty} \int \ldots \int I_{B^{*}}\left(x_{1}\right) p\left(t / n ; x_{1}, x_{2}\right) I_{A^{*}}\left(x_{2}\right) \ldots p\left(t / n ; x_{n-1}, x_{n}\right) I_{A^{*}}\left(x_{n}\right) d x_{1} \ldots \\
& =\int I_{B^{*}}(x) \tilde{p}_{A^{*}}(t, x, y) I_{A^{*}}(y) d y d x \\
& =\int_{B^{*}} \tilde{u}_{A^{*}}(t, x) d x
\end{aligned}
$$

where we have used (3.3) in the first and last lines, the definition of $\tilde{p}_{A}(t ; x, y)$ in the second and fourth lines, and Theorem 2 in the third line. We conclude with (3.5) that

$$
\int_{B} u_{A}(t, x) d x \leq \int_{B^{*}} u_{A^{*}}(t, x) d x .
$$

By Lemma 3.1, this proves (1.6). Inequality (1.7) follows by setting $F(y)=y^{p}$ in (1.6) to obtain

$$
\left\|u_{A}(t, \cdot)\right\|_{p} \leq\left\|u_{A^{*}}(t, \cdot)\right\|_{p} \quad(1 \leq p<\infty)
$$

and then letting $p \rightarrow \infty$.

To discuss the cases of equality in (1.6), suppose that $F \circ u_{A^{*}}(t, \cdot)$ is non-constant, and assume for the moment that $A$ satisfies condition (2.20). Since $A^{\sigma}$ and $B^{\sigma}$ are equimeasurable with $A$ and $B$, inequality (3.18) implies that

$$
\int_{B^{*}} u_{A^{*}}(t, x) d x-\int_{B} u_{A}(t, x) d x \geq \sup _{\sigma} \int_{B^{\sigma}} u_{A^{\sigma}}(t, x) d x-\int_{B} u_{A}(t, x) d x .
$$


If $A$ differs from every disc by a set of positive volume, then, by Lemma 2.8, there exists a reflection $\sigma$ so that $A^{\sigma}$ differs from both $A$ and $\sigma A$ by a set of positive volume. Propositions 2.1 and 2.3 imply that

$$
\int_{B^{\sigma}} u_{A^{\sigma}}(t, x) d x-\int_{B} u_{A}(t, x) d x \geq \int_{B} \int \mathcal{W}_{x, y}^{t}\left(E^{t}\right) d y d x>0
$$

for any Borel set $B \subset A$ of positive volume, so the right hand side of (3.21) is positive. By Lemma 3.1, inequality (1.6) is strict. Inequality (1.6) is also strict if $A$ differs from a disc $D$ by a set of zero volume but $D \backslash A$ is not polar, since a path in $D$ has positive probability of hitting $D \backslash A$ before leaving $D$. This settles the case where (2.20) holds.

On the other hand, if (2.20) does not hold, we can write $A=A_{1} \cup A_{2}$, where $A_{1}$ and $A_{2}$ are disjoint, both have positive volume, and

$$
\int_{A_{1}} \int_{A_{2}} p_{A}(t ; x, y) d x d y=0 .
$$

Then

$$
\begin{aligned}
\int_{A} F\left(u_{A}(t ; x)\right) d x & =\int_{A_{1}} F\left(u_{A_{1}}(t ; x)\right) d x+\int_{A_{2}} F\left(u_{A_{2}}(t ; x)\right) d x \\
& \leq \int_{A_{1}^{*}} F\left(u_{A_{1}^{*}}(t ; x)\right) d x+\int_{A_{2}^{*}} F\left(u_{A_{2}^{*}}(t ; x)\right) d x \\
& <\int_{A^{*}} u_{A^{*}}(t ; x) d x .
\end{aligned}
$$

Here, the first line follows from (3.22), the second follows from (1.6), and the last line follows since the disjoint union of two discs cannot produce equality in (1.6): Position the two discs so that they touch but their interiors do not intersect, and then perform a two-point rearrangement such that the resulting $A^{\sigma}$ is connected. We conclude again that (1.6) is strict.

For the analysis of the cases of equality in (1.7) we use (2.10) of Proposition 2.1 (with $B=\{x\}$, $C=A)$ in place of (2.6).

\subsection{Some applications}

We briefly indicate how Theorem 1 and the rearrangement methods used in the proof can yield inequalities for exit time moments and the spectrum of Dirichlet heat kernels.

Corollary 3.2 [39] (Moment inequalities) Let $A \subset \mathbb{M}_{\kappa}$ be a Borel set of finite volume. Then the moments of the exit time from A defined by

$$
\mathcal{M}_{p, n}(A)=\left\|\mathbf{E}_{x}\left[T_{A}^{n}\right]\right\|_{p}
$$

satisfy

$$
\mathcal{M}_{p, n}(A) \leq \mathcal{M}_{p, n}\left(A^{*}\right)
$$

for all $1 \leq p \leq \infty$, and $n>0$. Equality in (3.27) for some value of $p$ and $n$ implies that there is a disc $D$ so that $A \backslash D$ has zero volume and $D \backslash A$ is polar. 
Proof: We will show that, if $F$ is nonnegative and convex with $F(0)=0$ and $G$ is nonnegative and increasing with $G(0)=0$, then

$$
\int F\left(\mathbf{E}_{x}\left[G\left(T_{A}\right)\right]\right) d x \leq \int F\left(\mathbf{E}_{x}\left[G\left(T_{A^{*}}\right)\right]\right) d x
$$

Setting $F(y)=y^{p}$ and $G(T)=T^{n}$ yields (3.27).

By Lemma 3.1, the proof of (3.28) amounts to showing that

$$
\int_{B} \mathbf{E}_{x}\left[G\left(T_{A}\right)\right] d x \leq \int_{B^{*}} \mathbf{E}_{x}\left[G\left(T_{A^{*}}\right)\right] d x
$$

for all Borel sets $B \subset A$. We write

$$
\mathbf{E}_{x}\left[G\left(T_{A}\right)\right]=\int_{0}^{\infty} \mathbf{P}_{x}\left[G\left(T_{A}\right)>t\right] d t=\int_{0}^{\infty} \mathbf{P}_{x}\left[T_{A}>g(t)\right] d t=\int_{0}^{\infty} u(g(t), x) d t
$$

where $g(t)$ is the inverse function of $G$ and $u(t, x)$ is the distribution function of the exit time. With Fubini's theorem we see that

$$
\int_{B} \mathbf{E}_{x}\left[G\left(T_{A}\right)\right] d x=\int_{0}^{\infty} \int_{B} u(g(t), x) d x d t
$$

Applying Theorem 1 to the inner integral completes the proof.

The next inequality is a by-product of the proof, rather than the statement of Theorem 1 .

Corollary 3.3 Let $A \subset \mathbb{M}_{\kappa}$ be a Borel set of finite volume. Then

$$
\int f P_{A}^{t} g \leq \int f^{*} P_{A^{*}}^{t} g^{*}
$$

for any pair of nonnegative measurable functions $f$ and $g$ which vanish at infinity.

If A neither $f$ nor $g$ vanish almost everywhere, then equality occurs only when there is a disc $D$ so that $A \backslash D$ has zero volume, $D \backslash A$ is polar, and the restrictions of $f$ and $g$ to $D$ coincide a.e. with nondecreasing functions of the distance to the center of $D$.

PROOF: For characteristic functions $f=I_{B}, g=I_{C}$ the claim is obtained by replacing $u_{A}(t, x)$ with $P_{A}^{t} I_{C}$ (and correspondingly $\tilde{u}_{A}$ with $\tilde{P}^{t} I_{A^{c}}$ ) in the proof of Theorem 1 . The general claim (3.32) follows by writing $f$ and $g$ with the layer-cake principle as

$$
f(x)=\int_{0}^{\infty} I_{f(x)>s} d s, \quad g(x)=\int_{0}^{\infty} I_{g(x)>s} d s
$$


Corollary 3.3 immediately implies the Faber-Krahn inequality: Let $\lambda_{1}(A)$ and $\lambda_{1}\left(A^{*}\right)$ be the first Dirichlet eigenvalues of $A$ and $A^{*}$, respectively, and let $\phi_{A}$ and $\phi_{A^{*}}$ be the corresponding eigenfunctions. Then

$$
e^{-\lambda_{1}(A)}=\int \phi_{A}\left(P_{A}^{t} \phi_{A}\right) d x \leq \int\left(\phi_{A}\right)^{*} P_{A^{*}}^{t}\left(\phi_{A}\right)^{*} d x \leq e^{-\lambda_{1}\left(A^{*}\right)},
$$

where the first equation is the definition of $\phi_{A}$, the second is (3.32), and the third uses the variational characterization of $\lambda_{1}\left(A^{*}\right)$. Equality occurs only when $A$ is essentially a disc.

As in the proof of Theorem 1, one can combine Propositions 2.1 and 2.3 with Lemma 2.8 to estimate $\lambda_{1}(A)-\lambda_{1}\left(A^{*}\right)$ from below. If (2.20) holds for $A$, and $A^{\sigma}$ differs from both $A$ and $A^{\sigma}$ by a set of positive measure, then for large $t$, the event $E^{t}$ occurs for most paths that remain in $A^{\sigma}$ up to time $t$. More precisely, a Borel-Cantelli argument implies that the integrals in (2.6) which involve $A$ decay at a faster exponential rate than the integrals which involve $A^{\sigma}$.

An estimate for the perimeter cannot be obtained in this way, since in general,

$$
\|\nabla f\|_{p}=\left\|\nabla f^{\sigma}\right\|_{p} \quad \text { and } \operatorname{Per}(A)=\operatorname{Per}\left(A^{\sigma}\right) .
$$

The reason is that $\left|\nabla f^{\sigma}\right|$ is equimeasurable with $|\nabla f|$ by construction. This implies, in particular, that the approximation of the spherically decreasing rearrangement by sequences of two-point rearrangements cannot converge in $W^{1, p}$.

Statements analogous to Theorem 1 hold for the trace of the heat kernel. We refrain from stating the most general results here and refer the interested reader to two recent papers of Morpurgo [40, 41].

Theorem 3.4 For any Borel set $A \subset \mathbb{M}_{\kappa}$ of finite positive measure,

$$
\operatorname{trace} P_{A}^{t} \leq \operatorname{trace} P_{A^{*}}^{t},
$$

with equality only if there is a disc $D$ so that $A \backslash D$ has zero volume, and $D \backslash A$ is polar.

As in the case of Theorem 1, inequality (3.36) is a direct consequence, via Trotter's formula (3.4), of Theorem 2). To analyze the cases of equality, note that

$$
\operatorname{trace} P_{A^{*}}^{t}-\operatorname{trace} P_{A}^{t} \geq \sup _{\sigma} \operatorname{trace} P_{A^{\sigma}}^{t}-\operatorname{trace} P_{A}^{t},
$$

since $A^{\sigma}$ is equimeasurable with $A$. The right side of (3.37) can be estimated by modifying the map $L$ in the proof of Proposition 2.1 so that it takes closed loops centered at a point $x$ to closed loops centered at $x$ or $\sigma x$. This shows that

$$
\sum_{ \pm} I_{B^{\sigma}}\left(x^{ \pm}\right) p_{A^{\sigma}}\left(t ; x^{ \pm}, x^{ \pm}\right)-I_{B}\left(x^{ \pm}\right) p_{A}\left(t ; x^{ \pm}, x^{ \pm}\right)=\sum_{ \pm} I_{B^{\sigma}}\left(x^{ \pm}\right) \mathcal{W}_{x^{ \pm}, x^{ \pm}}^{t}
$$

where $E^{t}$ is the event defined in Proposition 2.1. Integrating over $B=A$ results in

$$
\operatorname{trace} P_{A^{\sigma}}^{t}-\operatorname{trace} P_{A}^{t}=\int \mathcal{W}_{x, x}^{t}\left(E^{t}\right) d x
$$


which is the analogue of Proposition 2.1. Under the additional assumption (2.20), the right hand side of (3.39) vanishes only when $A$ coincides with either $A^{\sigma}$ or $\sigma A^{\sigma}$ (up to a set of zero volume). We conclude as in the proof of Theorem 1 that equality occurs only when $A$ is essentially a disc.

We add a corollary of (2.10) and (3.38) which was suggested to us by Laugesen [35].

Corollary 3.5 Let $D$ be a disc in $\mathbb{M}_{\kappa}$, centered at $x^{*}$, with $D \neq \mathbb{M}_{\kappa}$. Then the functions $u_{D}(t, x)$ and $p_{D}(t ; x, x)$ are strictly decreasing functions of $d\left(x, x^{*}\right)$ for $t>0, x \in D$.

PROOF: By symmetry, both $p_{D}(t ; x, x)$ and $u_{D}(t ; x)$ depend only on $t$ and $d\left(x, x^{*}\right)$. Let $x$ and $z$ be two points in $D$ with $d\left(x, x^{*}\right)<d\left(z, x^{*}\right)$. We want to show that

$$
\begin{aligned}
u_{D}(t, z) & <u_{D}(t, x), \quad \text { and } \\
p_{D}(t ; z, z) & <p_{D}(t ; x, x),
\end{aligned}
$$

Assume without loss of generality that $x$ lies on the shortest geodesic that connects $z$ with $x^{*}$. Let $\sigma$ be the reflection which maps $x$ to $z$, let $H^{o}$ be the hypersurface of fixed points of $\sigma$, and let $H^{+}$be the half-space containing $x^{*}$ and $x$. Clearly, $D^{\sigma}=D$. To see the first inequality, choose $A=C=D$ and $B=\{z\}$ in (2.10) and integrate over $y \in D$. Similarly, setting $A=D$ and $B=C=\{z\}$ in (3.38) implies (3.41).

\section{Perimeter and heat flow}

In this section we prove Theorem 3 and inequality (1.15). Since the right hand side of (1.15), which involves only the heat kernel on $\mathbb{M}$, is more manageable than the right hand side of (1.15), we prove Theorem 3 first.

\subsection{Definitions}

The perimeter of a set $A$ is the total variation of its characteristic function, that is,

$$
\operatorname{Per}(A):=\sup _{Y:|Y| \leq 1} \int_{A} \operatorname{div} Y(x) d x
$$

where the supremum is over all smooth compactly supported vector fields with $|Y| \leq 1$, and $\operatorname{div} Y$ denotes the divergence of $Y$ (see $[49,16]$ ).

In general, the inequality

$$
\operatorname{Per}(A) \leq \varliminf_{n \rightarrow \infty} \sup _{Y} \int g_{n}(x) \operatorname{div} Y(x) d x=\underline{\lim _{n \rightarrow \infty}}\left\|\nabla g_{n}\right\|_{1}
$$


holds for any sequence of smooth functions $g_{n}$ converging to $I_{A}$ in $L_{l o c}^{1}$. In particular, the perimeter is lower semicontinuous with respect to convergence in measure. If $A$ has finite perimeter, one can choose the approximating sequence $g_{n}$ so that

$$
\operatorname{Per}(A)=\lim _{n \rightarrow \infty}\left\|\nabla g_{n}\right\|_{1}
$$

and consequently

$$
\int_{\partial A}\langle\nu(x), Y(x)\rangle d S:=\lim _{n \rightarrow \infty} \int\left\langle-\nabla g_{n}(x), Y(x)\right\rangle d x
$$

exists for every smooth bounded vector field $Y$ on $M$.

If $A$ has smooth boundary, then (4.1) coincides with the definition of the perimeter as a surface integral. Furthermore, the right hand side of (4.4) coincides with the usual formula for the flux of $Y$ across the boundary of $A$, where $\nu(x)$ is the outward unit normal to the boundary of $A$ at a boundary point $x$, the pairing $\langle$,$\rangle denotes the inner product on the tangent space of M$ at $x$, and $d S$ is integration with respect to $(m-1)$-dimensional surface measure.

We will frequently use geodesic polar coordinates for computations (see $[42,20,11]$ for a more detailed description). Every point in a smooth complete Riemannian manifold $M$ can be represented as $x=\exp _{x_{o}}(r w)$, where $w$ is a unit tangent vector at $x_{o}$, the function $\exp _{x_{o}}$ is the exponential map from the tangent space of $M$ at $x_{o}$ into $M$, and $r=d\left(x, x_{o}\right)>0$. This representation is unique in a neighborhood of $x_{o}$, and we refer to $(r, w)$ as geodesic coordinates about $x_{o}$. Identifying the unit sphere in the tangent space of $M$ at $x_{o}$ with $\mathbb{S}^{m-1}$, we may express the Riemannian measure on $M$ in terms of Lebesgue measure on $\mathbb{R}_{+}$and the standard surface measure on $\mathbb{S}^{m-1}$ as $d x=\theta(r, w) d r d w$. The gradient and Laplacian of $\rho(x)=d\left(x, x_{o}\right)$ at a point $x=\exp _{x_{o}}(r w)$ can be written as

$$
\nabla \rho(x)=\partial_{r} \exp _{x_{o}}(r w), \quad \Delta \rho(x)=\partial_{r} \log \theta(r, w) .
$$

For the manifolds of constant curvature $\mathbb{M}_{\kappa}$, one can explicitly compute

$$
\theta_{\kappa}(r)=\left\{\begin{array}{cl}
\left(\frac{\sin (r \sqrt{\kappa})}{\sqrt{\kappa}}\right)^{m-1} & \text { if } \kappa>0 \\
r^{m-1} & \text { if } \kappa=0 \\
\left(\frac{\sinh (r \sqrt{-\kappa})}{\sqrt{-\kappa}}\right)^{m-1} & \text { if } \kappa<0
\end{array}\right.
$$

(see Theorem II.5.1 of [20].

Consider the Dirichlet problem (1.3)-(1.5) on a disc $D$ of radius $R$, centered at $x^{*}$ in $\mathbb{M}_{\kappa}$. By symmetry we can write the solution $u_{D}$ as

$$
u_{D}(t, x)=h_{\kappa}\left(t, d\left(x, x^{*}\right)\right),
$$

where $u_{D}(t, x)$ is defined by (1.2), the function $h_{\kappa}(t, r)$ is nonnegative and vanishes for $r \geq R$. By Corollary 3.5, $h_{\kappa}(t, r)$ is strictly decreasing in both $r$ and $t$ for $0<r<R$, and all $t>0$. 
Two classical inequalities relate the Laplacian on a complete smooth Riemannian manifold $M$ near a point $x_{o} \in M$ with the Laplacian on $\mathbb{M}_{\kappa}$. Let $A$ be a geodesic disc of radius $R$ centered at $x_{o}$ in $M$, and let $D$ be a disc of the same radius $R$ centered at $x^{*}$ in $\mathbb{M}_{\kappa}$. If $R$ is small enough we can use geodesic coordinates to define a diffeomorphism $\psi: A \rightarrow D$ by setting $\psi(x)=y$ when $x=\exp _{x_{o}}(r w)$ and $y=\exp _{x^{*}}(r w)$.

1. Günther's comparison theorem says that, if the sectional curvature of $M$ is bounded above by $\kappa$, then

$$
\partial_{r} \frac{\theta(r, w)}{\theta_{\kappa}(r)} \geq 0
$$

that is,

$$
\Delta \rho(x) \geq \Delta_{\kappa} \rho_{\kappa}(\psi(x)) .
$$

One implication is that the volume of $A$ is at least as large as the volume of $D$ [27] (see [20], III.2). Furthermore, $u_{D}(t, \psi(x))$ is a supersolution of (1.3)-(1.5) on $A$, that is

$$
u_{A}(t, x) \leq h_{\kappa}\left(t, d\left(x, x_{o}\right)\right)
$$

with equality only when $A$ is isometric to a disc in $\mathbb{M}_{\kappa}[38,22,31,42]$.

2. Bishop's comparison theorem asserts that the reverse inequalities hold if if the Ricci curvature of $M$ is bounded from below by $(m-1) \kappa$ :

$$
\partial_{r} \frac{\theta(r, w)}{\theta_{\kappa}(r)} \leq 0
$$

or, equivalently,

$$
\Delta \rho(x) \leq \Delta_{\kappa} \rho_{\kappa}(\psi(x)),
$$

where $\Delta_{\kappa}$ is the Laplacian on $\mathbb{M}_{\kappa}$ [11], Theorem 11.15 (see [20], III.3). Consequently, the volume of $A$ cannot exceed the volume of $D$, and

$$
u_{A}(t, x) \geq h_{\kappa}\left(t, d\left(x, x_{o}\right)\right)
$$

with equality only when $A$ is isometric to a disc in $\mathbb{M} \kappa$. [22, 31].

In other words, if $\kappa$ is a bound for the curvature of $M$, then the solution of the Dirichlet problem (1.3)-(1.5) on a small disc is bounded by the solution of the corresponding problem on a disc of the same radius in $\mathbb{M}_{\kappa}$. Analogous comparison results are known for the heat kernel (see [20], VIII.3).

\subsection{A simple perimeter estimate on spheres}

The following inequality will not be needed below. We present it here to introduce, in a simpler setting, the idea for the proof of Theorem 3. 
Proposition 4.1 Let $\mathbb{M}_{\kappa}$ be a sphere of constant curvature $\kappa>0$, and let $P_{\kappa}^{t}$ be the heat semigroup on $\mathbb{M}_{\kappa}$. For every $A \subset \mathbb{M}_{\kappa}$,

$$
\frac{1}{\operatorname{Per}(A)} \int_{A^{c}} P_{\kappa}^{t} I_{A} d x \leq \frac{1}{\operatorname{Per}(H)} \int_{H^{c}} P_{\kappa}^{t} I_{H} d x
$$

where $H$ is a hemisphere.

REMARK Passing to the limit $t \rightarrow \infty$ in (4.14) results in

$$
\operatorname{Per}(A) \geq \frac{V(A) V\left(A^{c}\right)}{V(H)^{2}} \operatorname{Per}(H),
$$

where $V(A)$ denotes the volume of $A$, and correspondingly for $A^{c}$ and $H$. This inequality is related with Cheeger's inequality (see [20]). The right hand side of (4.15) is maximized for $V(A)=$ $V(H)=V\left(\mathbb{M}_{\kappa}\right) / 2$, when the statement becomes a special case of the classical isoperimetric inequality.

PROOF: By symmetry, the heat kernel on $\mathbb{M}_{\kappa}$ can be written in the form

$$
p_{\kappa}(t ; x, y)=q_{\kappa}(t, d(x, y)),
$$

where $q_{k}(t, r)$ is a strictly decreasing function of of $r$ for each fixed $t>0$. For any pair of smooth nonnegative functions $f$ and $g$ on $\mathbb{M}_{\kappa}$, we compute

$$
\begin{aligned}
\int_{\mathbb{M}_{\kappa}} f\left(P_{\kappa}^{t} g-g\right) d x & =\int_{0}^{t} \int_{\mathbb{M}_{\kappa}} f P_{\kappa}^{s} \Delta g d x d s \\
& =-\int_{0}^{t} \int_{\mathbb{M}_{\kappa}}\left\langle\nabla g, \nabla\left(P_{\kappa}^{s} f\right)\right\rangle d x d s \\
& =-\int_{0}^{t} \int_{\mathbb{M}_{\kappa}} \int_{\mathbb{M}_{\kappa}}\left\langle\nabla g(x), \nabla_{x} p_{\kappa}(s ; x, y)\right\rangle f(y) d y d x d s
\end{aligned}
$$

where $\langle\cdot, \cdot\rangle$ denotes the inner product on the tangent space of $M_{\kappa}$ at $x$. We have used the heat equation in (4.17), integrated by parts in (4.18), and spelled out the heat kernel in (4.19). Setting $f=I_{A^{c}}$ yields

$$
\int_{A^{c}} P_{\kappa}^{t} g-g d x=-\int_{0}^{t} \int_{\mathbb{M}_{\kappa}} \int_{A^{c}}\left\langle\nabla g(x), \nabla_{x} p_{\kappa}(s ; x, y)\right\rangle d y d x d s .
$$

For fixed $s>0$ and $x \in \mathbb{M}_{\kappa}$, the inner integral can be estimated by taking the negative part of the integrand,

$$
\begin{aligned}
-\int_{A^{c}}\left\langle\nabla g(x), \nabla_{x} p_{\kappa}(s ; x, y)\right\rangle d y \\
\leq \int_{\mathbb{M}_{\kappa}}\left\langle\nabla g(x), \nabla_{x} p_{\kappa}(s ; x, y)\right\rangle_{-} d y \\
\quad=\int_{S^{m-1}}\langle\nabla g(x), w\rangle_{+} d w \int_{0}^{\infty}\left|\partial_{r} q_{\kappa}(s ; r)\right| \theta_{\kappa}(r) d r
\end{aligned}
$$


We have switched to geodesic coordinates $y=\exp _{x}(r, w)$, computed $\nabla_{x} d(x, y)=-w$, and used the fact that $\theta_{\kappa}$ does not depend on the direction vector $\omega$ to separate the integrals. Clearly, the right hand side of (4.22) is a constant multiple of $|\nabla g(x)|$, where the constant depends only on $s$, the dimension $m$, and the curvature $\kappa$. Performing the integrations over $x$ and $s$ in (4.20), we obtain

$$
\int_{A^{c}} P_{\kappa}^{t} g-g d x \leq C(t, m, \kappa)\|\nabla g\|_{1} .
$$

Using an approximating sequence as in (4.3), we conclude that

$$
\int_{A^{c}} P_{\kappa}^{t} I_{A} d x \leq C(t, m, \kappa) \operatorname{Per}(A) .
$$

We claim that for a hemisphere $H$, inequality (4.24) holds with equality. Inserting an approximating sequence in (4.20), taking limits, and using (4.4), we see that

$$
\int_{H^{c}} P_{\kappa}^{t} I_{H} d x=\int_{0}^{t} \int_{\partial H} \int_{H^{c}}\left\langle\nu(x), \nabla_{x} p_{\kappa}(s ; x, y)\right\rangle d y d x d s
$$

where $\nu(x)$ is the outward normal to $\partial H$ at $x$. It is a special property of the hemisphere that the outward normal $\nu(x)$ at a point $x \in \partial H$ forms an acute angle with the shortest geodesic from $x$ to $y$, if and only if $y \in H^{c}$. Since $p_{\kappa}(s ; x, y)$ increases along that geodesic, it follows that for $x \in \partial H$,

$$
\int_{H^{c}}\left\langle\nu(x), \nabla_{x} p_{\kappa}(s ; x, y)\right\rangle d y=\int_{S^{m-1}}\langle\nu(x), w\rangle_{+} d w \int_{0}^{\infty}\left|\partial_{r} q_{\kappa}(s ; r)\right| \theta_{\kappa}(r) d r .
$$

Integrating over $y$ and $s$ in (4.25) we see that $H$ satisfies (4.24) with equality. This completes the proof.

\subsection{Proof of Theorem 3}

The special case of inequality (1.19) in $\mathbb{R}^{m}$ can be obtained by computing the constant $C(t, m, 0)$ in (4.23) and studying its asymptotics as $t \rightarrow 0$. On a general manifold, such a computation has to be replaced by estimates, as explicit formulas for the heat kernel are not available. We approximate the heat kernel $p(t ; x, y)$ on $M$ by $q(t ; d(x, y))$, where

$$
q(t ; r)=\frac{1}{(4 \pi t)^{m / 2}} e^{-r^{2} /(4 t)}
$$

is the function defining the heat kernel on $\mathbb{R}^{m}$.

The integral operators $Q^{t}$ given by the kernels $q(t ; d(x, y))$ can be used to construct the heat kernel on $M$ exactly (see, for example, $[10,45]$ ). Here, we mainly need that each $Q^{t}$ is a bounded linear operator on $L^{p}$ and that $Q^{t} f \rightarrow f$ as $t \rightarrow 0$ pointwise at least for smooth functions $f$ with 
compact support. Another useful observation is that for $R>0$ and $t$ sufficiently small, $q(t ; d(x, y))$ is concentrated on the diagonal in the sense that the contribution of

$$
\iint_{d(x, y) \geq R} f(x) q(t ; d(x, y)) g(y) d x d y \leq\|f\|_{\infty}\|g\|_{1} q(t ; R)
$$

is exponentially small in $t^{-1}$ as $t \rightarrow 0$, uniformly for $R$ bounded away from zero. The next lemma shows that $Q^{t} f$ almost solves the heat equation.

Lemma 4.2 Assume that the Ricci curvature of $M$ is bounded below, and the sectional curvature is bounded above. Then there exists a constant $C$ (depending only on $M$ ) such that for $t \leq 1$ :

$$
\left\|\left(\partial_{t}-\Delta\right) Q^{t} f\right\|_{\infty} \leq C\|f\|_{\infty}
$$

ProOF: Let $\rho(x)=d(x, y)$, fix $y$, and let $w$ be a unit vector. Using geodesic coordinates $x=$ $\exp _{y}(\rho w)$ about $y$, we calculate

$$
\left(\partial_{t}-\Delta\right) q(t ; \rho(x))=\frac{\rho(x)}{2 t}\left(\Delta \rho(x)-\frac{(m-1)}{\rho(x)}\right) q(t ; \rho(x)) .
$$

It is easy to see from the explicit formulas in (4.6) that on $\mathbb{M}_{\kappa}$,

$$
\left|\Delta_{\kappa} \rho(x)-\frac{m-1}{\rho(x)}\right| \leq C(\kappa) \rho(x) .
$$

By our assumptions on the curvature and the inequalities (4.12) and (4.9) of Bishop and Günther, a statement of the form (4.31) holds also on $M$, and we conclude that

$$
\left|\left(\partial_{t}-\Delta\right) q(t ; \rho(x))\right| \leq C(M) \frac{\rho(x)^{2}}{t} q(t ; \rho(x))
$$

for $x$ in a neighborhood of $y$ where geodesic coordinates are valid. It follows that

$$
\left|\left(\partial_{t}-\Delta\right) Q^{t} f\right|(x) \leq C(M)\|f\|_{\infty} \int_{\mathbb{S} m-1} \int_{0}^{R(y, w)} \frac{r^{2}}{t} q(t ; r) \theta(r, w) d r d w,
$$

where $R(y, w)$ denotes the maximal length of a distance-minimizing geodesic starting at $y$ in direction $w$. We have used the fact that in a complete manifold, the cut locus has zero volume. We claim that the last integral is finite. Indeed, using Bishop's inequality (4.11), we see that for $y \in M$ and $w \in \mathbb{S}^{m-1}$,

$$
\begin{aligned}
\int_{0}^{R(y, w)} \frac{r^{2}}{t} q(t ; r) \theta(r, w) d r & \leq \int_{0}^{\infty} \frac{r^{2}}{t} q(t ; r) \theta_{\kappa}(r) d r \\
& =\int_{0}^{\infty} q(1, r) \frac{r^{2} \theta_{\kappa}(r \sqrt{t})}{t^{(m+1) / 2}} d r
\end{aligned}
$$


where $\kappa$ is determined by the lower bound on the Ricci curvature of $M$. Since the right hand side of (4.35) is an increasing function of $t$ and takes a finite value for $t=1$, it is uniformly bounded for $t \leq 1$.

Inequality (4.29) implies a corresponding bound for the difference between $Q^{t}$ and the true heat kernel $P^{t}$ :

Lemma 4.3 Under the assumptions of Lemma 4.2, we have for $t \leq 1$

$$
\left\|\left(Q^{t}-P^{t}\right) f\right\|_{\infty} \leq C\|f\|_{\infty} t
$$

where $C$ is the constant of Lemma 4.2 .

PROOF: By Lemma 4.2, the function

$$
v(t, x)=\left(P^{t}-Q^{t}\right) f(x)-C\|f\|_{\infty} t
$$

satisfies

$$
\left(\partial_{t}-\Delta\right) v(t, x) \leq 0 \quad(t \leq 1)
$$

Since $v(0, x)=0$, we conclude by the maximum principle that $v(t, x) \leq 0$. Repeating the argument for

$$
\tilde{v}(t, x)=-\left(P^{t}-Q^{t}\right) f(x)-C\|f\|_{\infty} t
$$

gives the claim.

Inequality (1.19) holds with $P^{t}$ replaced by $Q^{t}$ :

Lemma 4.4 If $A \subset M$ is a bounded set with finite perimeter, then

$$
\operatorname{Per}(A) \geq \varlimsup_{t \rightarrow 0^{+}} \sqrt{\frac{\pi}{t}} \int_{A^{c}} Q^{t} I_{A} d x
$$

PROOF: Lemma 4.2 implies that for any two smooth nonnegative functions $f$ and $g$ with compact support,

$$
\begin{aligned}
\int f\left(Q^{t} g-g\right) d x & =\int_{0}^{t} \int_{M} f \partial_{s} Q^{s} g d x d s \\
& =\int_{0}^{t} \int_{M} f \Delta\left(Q^{s} g\right) d x d s+\|f\|_{\infty}\|g\|_{1} \mathrm{O}(t) \quad(t \rightarrow 0) .
\end{aligned}
$$

Hence we may repeat the computation in (4.17)-(4.20) to obtain

$$
\int_{A^{c}}\left(Q^{t} g-g\right) d x=-\int_{0}^{t} \int_{M} \int_{A^{c}}\left\langle\nabla g(x), \nabla_{x} q(s ; d(x, y))\right\rangle d y d x d s+\|g\|_{1} \mathrm{O}(t) .
$$


The inner integral on the right hand side can be estimated as in the proof of Proposition 4.1

$$
\begin{aligned}
-\int_{A^{c}}\left\langle\nabla g(x), \nabla_{x} q\right. & (s ; d(x, y))\rangle d y \\
\leq & \int_{M}\left\langle\nabla g(x), \nabla_{x} q(s ; d(x, y))\right\rangle_{-} d y \\
& =\int_{S^{m-1}}\langle\nabla g(x), w\rangle_{+} \int_{0}^{R(x, w)}\left|\partial_{r} q(s ; r)\right| \theta(r, w) d r d w .
\end{aligned}
$$

In the first step, we have taken the negative part of the integrand. In the second step, we have switched to geodesic coordinates $y=\exp _{x}(r, w)$; here $R(x, w)$ is the maximal length of a distanceminimizing geodesic starting at $x$ in direction $w$. Since $g$ is compactly supported, we have

$$
R_{o}=\inf _{x \in \operatorname{supp} g} \inf _{w \in \mathbb{S}^{m-1}} R(x, w)>0 .
$$

For given $\varepsilon>0$, there exists a positive number $R_{\varepsilon} \in\left(0, R_{o}\right)$ such that $\left|\theta(r, w)-r^{m-1}\right| \leq \varepsilon$ for all $r<R_{\varepsilon}$ and all $\omega \in S^{m-1}$. On the other hand, using Bishop's inequality (4.11) again, we see that

$$
\begin{aligned}
\int_{\left[R_{\varepsilon}, R(x, r)\right]}\left|\partial_{r} q(s ; r)\right| \theta(r, w) d r & \leq \int_{R_{\varepsilon}}^{\infty} \frac{r}{2 s} q(s ; r) \theta_{\kappa}(r) d r \\
& \leq \frac{1}{2 \sqrt{s}} \int_{R_{\varepsilon} / \sqrt{s}}^{\infty} q(1, r) r \theta_{-|\kappa|}(r) d r
\end{aligned}
$$

which is exponentially small in $s^{-1}$ as $s \rightarrow 0$. It follows that

$$
\begin{aligned}
\int_{0}^{R(x, w)}\left|\partial_{r} q(s ; r)\right| \theta(r, w) d r & =\sqrt{\frac{\pi}{s}} \int_{0}^{\infty}(4 \pi s)^{-(m+1) / 2} e^{-r^{2} / 4 s} r^{m} d r(1+o(1)) \\
& =\sqrt{\frac{\pi}{s}}\left((m+1) \omega_{m+1}\right)^{-1}(1+o(1))
\end{aligned}
$$

uniformly for $x$ in the support of $g$ and $w \in S^{m-1}$. The value of the spherical integral on the right hand side of (4.44) is easily computed as

$$
\int_{\mathbb{S}^{m-1}}\langle\nabla g(x), w\rangle_{+} d w=\omega_{m-1}|\nabla g(x)|
$$

where $\omega_{m-1}$ is the volume of the unit disc in dimension $m-1$. Combining equations (4.49) and (4.50) with (4.44) yields

$$
-\int_{A^{c}}\left\langle\nabla g(x), \nabla_{x} q(s ; d(x, y))\right\rangle d y \leq \sqrt{\frac{\pi}{s}} \frac{\omega_{m-1}}{(m+1) \omega_{m+1}}|\nabla g(x)|(1+o(1))
$$

We perform the integrations over $x$ and $s$ in (4.42) to obtain

$$
\begin{aligned}
\lim _{t \rightarrow 0^{+}} \sqrt{\frac{\pi}{t}} \int_{A^{c}} & Q^{t} g(x) d x \\
& \leq \pi \frac{\omega_{m-1}}{(m+1) \omega_{m+1}} \int_{M}|\nabla g(x)| d x \lim _{t \rightarrow 0^{+}} \frac{1}{\sqrt{t}} \int_{0}^{t} \frac{1}{\sqrt{s}}(1+o(1)) d s \\
& =\|\nabla g\|_{1}
\end{aligned}
$$


The claim follows by approximating $I_{A}$ with a suitable sequence of smooth functions and applying (4.4).

Lemma 4.5 If the boundary of A is twice continuously differentiable, then the limit in (4.39) exists, and the inequality holds with equality.

PROOF: We approximate $I_{A}$ with a sequence of smooth compactly supported functions $g_{n}$ as in (4.3), and use equations (4.40)-(4.42) from the proof of Lemma 4.4). Taking the limit $n \rightarrow \infty$ yields, with (4.4),

$$
\int_{A^{c}} Q^{t} I_{A} d x=\int_{0}^{t} \int_{\partial A} \int_{A^{c}}\left\langle\nu(x), \nabla_{x} q(s ; d(x, y))\right\rangle d y d x d s+\|g\|_{1} \mathrm{O}(t),
$$

where $\nu$ is the outward unit normal to $A$ at the boundary point $x$. Similarly, (4.43) becomes

$$
\int_{A^{c}}\left\langle\nu(x), \nabla_{x} q(s ; d(x, y))\right\rangle d y \leq \int_{\mathbb{M}}\left\langle\nu(x), \nabla_{x} q(s ; d(x, y))\right\rangle_{+} d y .
$$

We need to estimate the difference between the two sides of (4.56). Consider first, for given $x \in \partial A$, the set of points $y \in A^{c}$ where the tangent to the shortest geodesic from $x$ to $y$ at $x$ forms an obtuse angle with $\nu(x)$ (i.e., it points into $A$ ). The smoothness and compactness of $\partial A$ imply that for $x \in \partial A$, the $(m-1)$-dimensional surface measure of the set

$$
S_{-}(x, r)=\left\{w \in \mathbb{S}^{m-1} \mid \exp _{x}(r w) \in A^{c},\langle\nu(x), w\rangle<0\right\}
$$

is of the order $r^{2}$ uniformly in $x \in \partial A$, which shows that

$$
\begin{aligned}
\int_{A^{c}}\left\langle\nu(x), \nabla_{x} q(s ; d(x, y))\right\rangle_{-} d y & \leq \int_{0}^{\infty} \frac{r}{2 s} q(s ; r) \theta_{\kappa}(r) \int_{S_{-}(x, r)}\langle w, \nu(x)\rangle_{+} d w d r \\
& \leq \text { Const. } \int_{0}^{\infty} \frac{r^{3}}{2 s} q(s ; r) \theta_{\kappa}(r) d r \\
& =\mathrm{O}(\sqrt{s}) .
\end{aligned}
$$

Similar considerations for the set of points $y \in A$ where the shortest geodesic from $x$ and $y$ forms an acute angle with the normal $\nu(x)$ show that

$$
\int_{A}\left\langle\nabla_{x} q(s ; d(x, y)), \nu(x)\right\rangle_{+} d y=\mathrm{O}(\sqrt{s})
$$

uniformly for $x \in \partial A$. Thus, the difference between the two sides of (4.56) is of order $\mathrm{O}(\sqrt{s})$. The claim follows by integrating over $x \in \partial A$ and $s \in[0, t]$.

Proof of TheOREM 3 By Lemma 4.3,

$$
\int_{A^{c}}\left(P^{t}-Q^{t}\right) I_{A} d x=\int_{A}\left(P^{t}-Q^{t}\right) I_{A^{c}} \leq C V(A) t
$$

for $0 \leq t \leq 1$, with a constant $C$ which depends only on $M$. Hence it is sufficient to prove the claims for $Q^{t}$ in place of $P^{t}$, which was done in Lemmas 4.4 and 4.5. 


\subsection{Proof of formula (1.15)}

As discussed in the introduction, (1.15) is related to Theorem 3 via the the approximation

$$
u_{A}(t, x) \approx I_{A} P^{t}\left(I_{A}-I_{A^{c}}\right)(x)=I_{A}\left(1-2 I_{A} P^{t} I_{A}\right)(x)
$$

(recall that $P^{t} I_{A}+P^{t} I_{A^{c}} \equiv 1$ ). It remains to prove that (4.60) is justified for sufficiently regular sets. By a theorem of Varadhan [50], the heat kernel on $M$ satisfies

$$
\lim _{t \rightarrow 0^{+}}-4 t \log p(t ; x, y)=d(x, y)^{2} .
$$

This suggests that both sides of (4.60) should be exponentially small in $\operatorname{dist}\left(x, A^{c}\right)^{2} / 4 t$ as $t \rightarrow 0$, which would justify (4.60) in the interior of $A$. Lemma 4.7 proved below contains a weaker statement which suffices for our purposes. We first consider the special case of a half-space.

Lemma 4.6 Let $H \subset \mathbb{M}_{\kappa}$ be a half-space. Then

$$
u_{H}(t, x)=I_{H} P^{t}\left(I_{H}-I_{H^{c}}\right),
$$

and the inequality

$$
1-u_{H}(t, x) \leq C_{1} e^{-\operatorname{dist}\left(x, H^{c}\right)^{2} / 4 t}+C_{2} t
$$

holds uniformly for $0 \leq t \leq 1, x \in H$.

PROOF: The two sides of (4.62) agree, since they solve the same Dirichlet problem on $H$.

For the second claim, we write

$$
\begin{aligned}
1-u_{H}(t, x) & =2 I_{H} P^{t} I_{H^{c}}(x) \\
& =2 I_{H} Q^{t} I_{H^{c}}(x)+O(t) \\
& \leq 2 \omega_{m-1} \int_{R}^{\infty} q(t, r) \theta_{\kappa}(r) d r+O(t)
\end{aligned}
$$

where we have used Lemma 4.3 in the second line, and replaced $H$ by a disc of radius $R=$ dist $\left(x, H^{c}\right)$ in the third. The last integral is estimated by

$$
\begin{aligned}
\int_{R}^{\infty} q(t, r) \theta_{\kappa}(r) d r & \leq \int_{R / \sqrt{t}}^{\infty} q(1, r)\left(\frac{r e^{-r \sqrt{|\kappa| t}}}{\sqrt{t}}\right)^{m-1} d r \\
& \leq C(m, \kappa) q(1, R / \sqrt{t}),
\end{aligned}
$$

where we have used (4.6) in the first line, and used that the integrand increases with $t$ in the second. Combining (4.66) and (4.68) gives the claim. 
Lemma 4.7 Assume that the Ricci curvature of $M$ is bounded below by $(m-1) \kappa($ where $m$ is the dimension of $M)$, and let $A \subset M$ be a Borel set. Then for every fixed $R_{o}>0$, there exist constants $C_{1}, C_{2}, C_{3}$ such that

$$
P^{t} I_{A^{c}}(x) \leq 1-u_{A}(t, x) \leq C_{1} e^{-\left(C_{2} \operatorname{dist}\left(x, A^{c}\right)\right)^{2} / 4 t}+C_{3} t
$$

holds for all $x$ with $\operatorname{dist}\left(x, A^{c}\right) \leq R_{o}$, and all $t>0$.

PROOF: The first inequality in (4.69) is just the fact that

$$
u_{A}(t, x) \leq P^{t} I_{A}(x) \leq 1
$$

To prove the second inequality, set $R=\operatorname{dist}\left(x, A^{c}\right)$. Clearly, replacing $A$ by a disc of radius $R$ about $x$ only decreases the exit time. By inequality (4.13),

$$
u_{A}(t, x) \geq u_{D}\left(t, x^{\prime}\right)
$$

where $D \subset \mathbb{M}_{\kappa}$ is a disc of radius $R$ centered at $x^{\prime}$, and $\kappa$ is determined by the lower bound on the Ricci curvature.

Next, we choose a collection of $m+1$ half-spaces $H_{i} \subset \mathbb{M}_{\kappa}$ such that their intersection contains $x^{\prime}$ and is contained in $D$. Then

$$
1-u_{D}\left(t, x^{\prime}\right) \leq \sum_{i}\left(1-u_{H_{i}}\right)\left(t, x^{\prime}\right)
$$

It can be arranged that dist $\left(x^{\prime}, H_{i}^{c}\right) \geq C R$, where the constant depends on $\kappa, m$, and (in case $\kappa<0$ ) on $R_{o}$. We complete the proof by combining inequalities (4.71) and (4.72) with Lemma 4.6 and adjusting the constants.

The next step is an estimate near the boundary.

Lemma 4.8 Assume that the boundary of $A \subset M$ is compact and twice continuously differentiable. Then there exists a constant $C$ such that

$$
\sup _{x \in A}\left|P^{t}\left(I_{A}-I_{A^{c}}\right)-u(t, x)\right| \leq C \sqrt{t}
$$

for all $t>0$.

PROOF: The restriction of

$$
v(t, x)=P^{t}\left(I_{A}-I_{A^{c}}\right)-u(t, x)
$$

to $A$ satisfies the heat equation on $A$, with zero initial values, and boundary conditions

$$
v(t, x)=P^{t}\left(I_{A}-I_{A^{c}}\right)(x) \quad x \in \partial A .
$$


By the maximum principle, it suffices to prove that $v$ satisfies the claimed bound on the boundary of $A$. By Lemma 4.3 we may replace $P^{t}$ with $Q^{t}$ in (4.75). We compute

$$
Q^{t}\left(I_{A}-I_{A^{c}}\right)=\int_{0}^{\infty} q(t, r) \int_{\mathbb{S}^{m-1}} I_{A}\left(\exp _{x}(r \omega)\right)-I_{A^{c}}\left(\exp _{x}(r \omega)\right) \theta(r, \omega) d \omega d r
$$

The inner integral on the right hand side is the difference between the areas occupied by $A$ and $A^{c}$, respectively in the geodesic sphere of radius $r$ about $x$. Since the boundary of $A$ is twice differentiable, the fraction of the area of the geodesic sphere of radius $r$ occupied by $A$ and $A^{c}$, respectively, $1 / 2+O(r)$. It is easy to compute from there that $v(t, x)=O(\sqrt{t})$ as $t \rightarrow 0$ uniformly for $t \leq 1$ and $x \in \partial A$.

Proposition 4.9 If $A \subset M$ is a bounded set with twice continuously differentiable boundary, then

$$
\operatorname{Per}(A)=\lim _{t \rightarrow 0^{+}} \sqrt{\frac{\pi}{4 t}} \int_{A} 1-u_{A}(t, x) d x
$$

PROOF: By Lemma 4.8, the function

$$
\sqrt{\frac{\pi}{4 t}}\left(1-u_{A}(t, x)-2 P^{t} I_{A^{c}}\right)
$$

is bounded uniformly for $0<t \leq 1$ and $x \in A$. By Lemma 4.7, it converges to zero pointwise almost everywhere as $t \rightarrow 0$. Lebesgue's dominated convergence theorem implies that

$$
\lim _{t \rightarrow 0^{+}} \sqrt{\frac{\pi}{4 t}} \int_{A} 1-u_{A}(t, x) d x=\lim _{t \rightarrow 0^{+}} \sqrt{\frac{\pi}{t}} \int_{A} P^{t} I_{A^{c}} d x .
$$

Due to the regularity assumptions on $A$, the limit on the right hand side exists and equals the perimeter of $A$.

\subsection{Concluding remarks}

We close by formulating two plausible generalizations of Theorems 1 and 2 to manifolds of nonconstant curvature. The first extends a long-standing conjecture due to Aubin [4] that on simply connected manifolds of non-positive curvature, the isoperimetric inequality should hold with the sharp Euclidean constants, with equality only for flat discs. While significant progress has been made in recent years $[23,33,19]$, the conjecture appears to be open for smooth manifolds of dimension larger than four. 
Conjecture 4.10 Suppose that $M$ is a simply connected, complete smooth manifold of non-positive sectional curvature. For a given $A \subset M$ of finite volume, let $A^{*}$ be the Euclidean disc centered at $x^{*}$ so that the Euclidean volume $V\left(A^{*}\right)$ agrees with the Riemannian volume $V(A)$. Then

$$
\int_{A} F\left(u_{A}(t, x)\right) d x \leq \int_{A^{*}} F\left(u_{A^{*}}(t, x)\right) d x \quad(t \geq 0)
$$

for all nonnegative convex functions $F$ with $F(0)=0$, and all $t \geq 0$. Furthermore,

$$
\int_{A} P^{t} I_{A} \leq \int_{A^{*}} Q^{t} I_{A^{*}} \quad(t \geq 0)
$$

where $Q^{t}$ is the Euclidean heat kernel.

By formula (1.15) and Theorem 3, either (4.80) or (4.81) would imply

$$
\operatorname{Per}(A) \geq \operatorname{Per}\left(A^{*}\right)
$$

which is the isoperimetric inequality conjectured by Aubin. The assumption that $M$ is simply connected is crucial, since otherwise $M$ may be compact, in which case $A=M, u_{M} \equiv 1, P^{t} I_{M} \equiv$ 1 , and $\operatorname{Per}(A)=0$ would contradict (4.80), (4.81), and (4.82). Note that in the special case where $A$ is a disc of radius $R$, the disc $A^{*}$ has larger radius than $A$ by Bishop's comparison theorem, and inequality (4.80) is weaker than (4.10).

Let now $M$ be a smooth compact $m$-dimensional Riemannian manifold whose Ricci curvature is bounded below by $(m-1) \kappa$, where $\kappa>0$, and set

$$
\beta=\frac{V(M)}{V_{\kappa}\left(\mathbb{M}_{\kappa}\right)} \leq 1
$$

where $V$ and $V_{\kappa}$ denote the Riemannian volumes on $M$ and $\mathbb{M}_{\kappa}$, respectively. For $A \subset M$, let $A^{*} \subset \mathbb{M}_{\kappa}$ be a disc with

$$
V(A)=\beta V_{\kappa}\left(A^{*}\right) .
$$

The Gromov-Levy isoperimetric inequality says that

$$
\operatorname{Per}(A) \geq \beta \operatorname{Per}\left(A^{*}\right)
$$

at least for sufficiently regular sets (see [20], IV.2, Remark 2).

Conjecture 4.11 Let $M$ be a compact smooth manifold whose Ricci curvature is bounded below by $(m-1) \kappa$ with $\kappa>0$, and let $A \subset M, \beta$, and $A^{*} \subset \mathbb{M}_{\kappa}$ as in (4.84). Then

$$
\int_{A} F\left(u_{A}(t, x)\right) d x \leq \beta \int_{A^{*}} F\left(u_{A^{*}}(t, x)\right) d x \quad(t \geq 0)
$$

for all nonnegative convex functions with $F(0)=0$, and

$$
\int_{A} P^{t} I_{A} \leq \beta \int_{A^{*}} P_{\kappa}^{t} I_{A^{*}} \quad(t \geq 0)
$$

where $P_{\kappa}^{t}$ denotes the heat kernel on $\mathbb{M}_{\kappa}$. 
Inequality (4.85) is contained in the $t \rightarrow 0$ asymptotics of either (4.86) or (4.87). Note that (4.86) and (4.87) are saturated for $t=0$ and in the limit $t \rightarrow \infty$ by definition of $\beta$. For $M=\mathbb{M}_{\kappa}$, we have $\beta=1$, so that (4.86) reduces to (1.6) of Theorem 1, and (4.87) reduces to (1.20). If $M$ is not isometric to $\mathbb{M}_{\kappa}$, then the Gromov-Levy inequality (4.85) is strict. In that case, formula (1.15) and Theorem 3 imply that Conjecture 4.11 holds at least for small values of $t$.

\section{Acknowledgments}

We wish to thank A. Baernstein, I. Herbst, C. Kerce, E. H. Lieb, M. Loss, R. S. Laugesen, P. McDonald, C. Morpurgo, and L. E. Thomas for many useful discussions, and R. Howard and M. Ledoux for valuable references. We especially thank M. Loss for initiating this collaboration by posing the motivating question and establishing the first contact between the authors. We also gratefully acknowledge the hospitality of MSRI during the Spring 1996 program on Convex Geometry and Geometric Functional Analysis. A.B.'s work was supported in part by National Science Foundation grant number DMS-9971493, and by a Sloan Fellowship.

\section{References}

[1] L. V. Ahlfors. Conformal Invariants: Topics in Geometric Function Theory. McGraw-Hill Series in Higher Mathematics, 1973.

[2] M. Aizenman and B. Simon. Brownian motion and Harnack's inequality for Schrödinger operators. Commun. Pure Appl. Math., 35:209-273, 1982.

[3] A. Alvino, G. Trombetti, and P.-L. Lions. On optimization problems with prescribed rearrangements. Nonlinear Anal., 13:185-220, 1989.

[4] T. Aubin. Problèmes isopérimétriques et espaces de Sobolev. J. Differential Geometry, 15:573-598, 1976.

[5] A. Baernstein and B. A. Taylor. Spherical rearrangements, subharmonic functions, and *functions in n-space. Duke Math. J., 43:245-268, 1976.

[6] C. Bandle. On symmetrization in parabolic equations. J. d'Analyse Mathémathique, 30:98$112,1976$.

[7] C. Bandle. Isoperimetric Inequalities and Applications. Pitman, Boston, 1986.

[8] W. Beckner. Sobolev inequalities, the Poisson semigroup, and analysis on the sphere $S^{n}$. Proc. Natl. Acad. Sci. USA, 89:4816-4819, 1985.

[9] Y. Benyamini. Two-point symmetrization, the isoperimetric inequality on the sphere and some applications. In Texas Functional Analysis Seminar 1983/84, Austin, Texas, pages 5376. Longhorn Notes, University of Texas Press, Austin, TX, 1984. 
[10] M. Berger, P. Gauduchon, and E. Mazet. Le spectre d'une variété riemannienne. Springer LNM 194, 1971.

[11] R. L. Bishop and R. J. Crittenden. Geometry of Manifolds. Academic Press, 1964.

[12] B. Bollobás and I. B. Leader. Compressions and isoperimetric inequalities. J. Combin. Theory Ser. A, 56:47-62, 1991.

[13] C. Borell. Geometric bounds on the Ornstein-Uhlenbeck velocity process. Z. Wahrsch. Verw. Gebiete, 70:227-237, 1985.

[14] H. J. Brascamp, E. H. Lieb, and J. M. Luttinger. A general rearrangement inequality for multiple integrals. J. Funct. Anal., 17:227-237, 1974.

[15] F. Brock and A. Yu. Solynin. An approach to symmetrization via polarization. Trans. Amer. Math. Soc., 352:1759-1796 (2000).

[16] Yu. D. Burago and V.A. Zalgaller. Geometric inequalities (English translation of the Russian original). Springer Grundlehren 285, 1980.

[17] A. Burchard. Cases of equality in the Riesz rearrangement inequality. Annals of Mathematics, 143:499-527, 1996.

[18] D. L. Burkholder. Exit time of Brownian Motion, Harmonic Majorization, and Hardy spaces. Advances in mathematics, 26:182-205, 1977.

[19] J. Cao. An isoperimetric comparison theorem for surfaces of variable curvature. In Proceedings of the Third Summer School on Differential Geometry, Partial Differential Equations and Numerical Analysis (in Spanish). Colec. Mem., 7, Acad. Colombiana Cienc. Exact. Fis. Natur., pages 1-7, Bogota, 1996.

[20] I. Chavel. Eigenvalues in Riemannian Geometry. Academic Press, 1984.

[21] J. Cheeger and S.-T. Yau. A lower bound for the heat kernel. Comm. Pure Appl. Math., 34:465-480, 1981.

[22] A. Debiard, B. Gaveau, and E. Mazet. Théorèmes de comparaison en géométrie riemannienne. Publ. Res. Inst. Math. Sci., 12:391-425, 1976/77.

[23] C. B. Croke. A sharp four-dimensional isoperimetric inequality. Comment. Math. Helvet., 59:187-192, 1984.

[24] M. Emery. Stochastic calculus in manifolds. Springer, 1990.

[25] P. Frankl and Z. Füredi. A short proof for a theorem of Harper about Hamming spheres. Discrete Math., 34:311-313, 1981. 
[26] R. Friedberg and J. M. Luttinger. Rearrangement inequalities for periodic functions. Arch. Rat. Mech. Anal., 61:35-44, 1976.

[27] P. Günther. Einige Sätze über das Volumenelement eines Riemannschen Raumes (German). Publ. Math. Debrecen, 7:78-93, 1960.

[28] K. Haliste. Estimates of harmonic measures. Ark. Math, 6:1-31, 1965.

[29] G. E. Hardy, J. E. Littlewood, and G. Pólya. Inequalities. Cambridge University Press, London and New York, 1952.

[30] I. Herbst and Z. Zhao. Sobolev spaces, Kac-regularity, and the Feynman-Kac formula. In Seminar on Stochastic Processes, 1987 (Princeton, NJ 1987), pages 171-191. Birkhäuser Boston, Boston, MA, 1988.

[31] N. Ikeda and S. Watanabe. Stochastic Differential Equations and Diffusion Processes. Second edition. North-Holland Publishing Co. Amsterdam - New York; Kodansha Ltd., Tokyo, 1989.

[32] T. Kato. Trotter's product formula for an arbitrary pair of self-adjoint contraction semigroups. In Topics in Functional Analysis (I. Gohberg and M. Kac, editors), pages 185-195, Academic Press, New York, 1978.

[33] B. Kleiner. An isoperimetric comparison theorem. Invent. Math., 108:37-47, 1992.

[34] R. Laugesen and C. Morpurgo. Extremals for eigenvalues of Laplacians under conformal mapping. J. Funct. Anal., 155:64-108, 1998.

[35] R. Laugesen. Personal communication, Summer 1997.

[36] M. Ledoux. Semigroup proofs of the isoperimetric inequality in Euclidean and Gauss space. Bull. Sci. Math., 118:485-510, 1994.

[37] E. H. Lieb. Existence and uniqueness of the minimizing solution to Chocquard's nonlinear equation. Studies in Applied Mathematics, 57:93-105, 1977.

[38] P. Malliavin. Asymptotics of the Green's function of a Riemannian manifold and Ito's stochastic integrals. Proc. Natl. Acad. Sci. USA 71:381-383 (1974).

[39] P. McDonald. Isoperimetric conditions, Poisson problems, and diffusion in Riemannian manifolds. preprint, 1999.

[40] C. Morpurgo. Sharp trace inequalities for intertwining operators on $S^{n}$ and $\mathbb{R}^{n}$. Intern. Math. Research Notices, 20:3565-3572, 1999.

[41] C. Morpurgo. Conformally invariant Sobolev inequalities and the spectrum of intertwining operators. Duke Math. J. (accepted) . 
[42] M. A. Pinsky. Stochastic Riemannian geometry. Probabilistic analysis and related topics, Vol. 1, pp. 199-236. Academic Press, New York, 1978.

[43] F. Pólya and M. Schiffer. Convexity of functionals by transplantation. J. Analyse Math., 3:245-345, 1953-54.

[44] F. Riesz. Sur une inégalité intégrale. J. London Math. Soc., 5:162-168, 1930.

[45] T. Sakai. Riemannian Geometry. AMS Translations of Mathematical Monographs, 149, 1996.

[46] J. Steiner. Einfacher Beweis der isoperimetrischen Hauptsätze. Crelle J. Reine Angew. Math., 18:281-296, 1838, and Ges. Werke, 2:77-91, Berlin 1882 (in German).

[47] D. W. Stroock. The Kac approach to potential theory, Part I. J. Math. Mech., 16:829-852, 1967.

[48] G. Talenti. Elliptic equations and rearrangements. Ann. Scuola Norm. Pisa Cl. Sci. (4), 3:697-718, 1976.

[49] G. Talenti. The standard isoperimetric theorem. Handbook of convex geometry, A:73-123, 1993.

[50] S.R.S. Varadhan. On the behavior of the fundamental solution of the heat equation with variable coefficients. Comm. Pure Appl. Math., 20:431-455, 1967.

[51] H. F. Weinberger. Symmetrization in uniformly elliptic problems. Studies in mathematical analysis and related topics, 424-428, 1962. Stanford Univ. Press, Stanford, Calif. 